IMPERIAL/TP/90-91/16

\title{
The Superparticle and the Lorentz Group
}

\author{
A.S. Galperin ${ }^{1 \dagger}$, P.S. Howe ${ }^{2}$ and K.S. Stelle ${ }^{1}$ \\ 1 The Blackett Laboratory, Imperial College, London SW7 2BZ \\ 2 Department of Mathematics, King's College, London WC2R 2LS
}

\begin{abstract}
We present a unified group-theoretical framework for superparticle theories. This explains the origin of the "twistor-like" variables that have been used in trading the superparticle's $\kappa$-symmetry for worldline supersymmetry. We show that these twistor-like variables naturally parametrise the coset space $\mathcal{G} / \mathcal{H}$, where $\mathcal{G}$ is the Lorentz group $S O^{\uparrow}(1, d-1)$ and $\mathcal{H}$ is its maximal subgroup. This space is a compact manifold, the sphere $S^{d-2}$. Our group-theoretical construction gives the proper covariantisation of a fixed light-cone frame and clarifies the relation between target-space and worldline supersymmetries.
\end{abstract}

\footnotetext{
$\dagger$ On leave from the Laboratory of Theoretical Physics, Joint Institute for Nuclear Research, Dubna, Head Post Office P.O. Box 79, 101000 Moscow USSR.
} 


\section{Introduction}

The Lorentz covariant formulation of the superstring theory and of its infinite tension limit - the massless relativistic superparticle - is a problem that has turned out to be more subtle than one might originally have expected. In special dimensions of the target spacetime ( $d=3,4,6$ and 10$)$, these theories describe equal number of bosonic and fermionic physical degrees of freedom. The main reason for this subtlety derives from the requirements of Lorentz invariance, which gives a much larger dimension to the spinor variable of the superstring/superparticle than to the bosonic coordinate. This is also a problem in supersymmetric field theories. The known ways of resolving this problem involve either auxiliary fields or the presence of a gauge symmetry to equalise the bosonic and fermionic physical degrees of freedom (or both). For the superstring/superparticle, this situation requires the existence of a non-trivial fermionic symmetry [1], which is generally called $\kappa$-symmetry. Generically, the $\kappa$-transformation for the spinor variable reads

$$
\delta \theta=\not p \kappa
$$

Unfortunately, this $\kappa$-symmetry has not yet found a completely satisfactory formulation in some of the most interesting cases. The principal difficulty can be easily understood: the parameter of the $\kappa$-transformations is a spinor of the same dimension as the spinor variable, but only a half of it contributes to the l.h.s. of (1.1) owing to the degeneracy of the momentum $\not p$ (on shell $p^{2}=\not p p=0$ ). Moreover, the $\kappa$-transformations only form a closed algebra subject to the theory's equations of motion. One well-known way out of these difficulties is to fix a light-cone frame, in which spinors become reducible representations of the surviving "little" group and the $\kappa$-parameter can be completely fixed. After this step, there are equal surviving numbers of bosonic and fermionic degrees of freedom. However, choosing the light-cone gauge destroys manifest Lorentz covariance. On the other hand, insisting on a Lorentz covariant quantization leads to the notorious "ghosts for ghosts" difficulty: in order to fix the irrelevant half of $\kappa$ one should introduce a spinor ghost variable, but this has twice as many components as one needs to compensate, so one half of it is in its own turn irrelevant and so on ad infinitum (see, for example [2] and refs therein).

Progress towards a resolution of these problems was made by Sorokin, Tkach and Volkov [3], who rewrote the superparticle action by introducing a commuting spinor variable and requiring a local worldline supersymmetry of the usual form. Upon use of the equations of motion, this local worldline supersymmetry can then be re-expressed as a $\kappa$-symmetry transformation.* The commuting spinor $\psi$ provides an algebraic solution to the particle's

* It is worthwhile to mention that the STV theory is different from the so-called spinning superparticle theory [4] : $i$ ) they describe different physical degrees of freedom, ii) the latter possesses both worldline supersymmetry and $\kappa$-symmetry, while in the former $\kappa$-symmetry is traded for the worldline supersymmetry. 
equation of motion $p^{2}=0$ through the relation $p^{m} \sim \bar{\psi} \gamma^{m} \psi$. In the cases of the $d=2+1, d=$ $3+1[3,5,6]$ and $d=5+1[5,6]$ superparticles, this approach yields a manifestly superPoincaré invariant formulation with all symmetries linearly realised (in fact, these actions possess a rigid superconformal invariance in the target superspace) and with a closed local worldline algebra.

In $d=9+1$, however, the problem has proved more subtle still. In ref. [7], an action for the $d=9+1$ superparticle was proposed that incorporates some of the ideas of ref. [3]. As in [3], the $p^{2}=0$ constraint is solved there by the introduction of a Majorana-Weyl commuting spinor, and after that the dynamical variables are redefined using this spinor. As in the $d=2+1,3+1 \& 5+1$ cases, this reformulation introduces a new "pregauge" symmetry that transforms the commuting spinor variable. In the $d=2+1,3+1 \& 5+1$ cases, these pregauge transformations are linear $Z_{2}, U(1), S U(2)$ transformations correspondingly, and are accompanied by a scale transformation; they all may be incorporated naturally into the local worldline supergroup. In the $d=9+1$ case, however, the pregauge transformation is non-linear: its parameter is a commuting Majorana-Weyl spinor, but only seven of its components are relevant. This parameter is determined up to a further gauge transformation with a vector parameter, and the latter is determined up to a scalar, so the transformation has $16-10+1=7$ independent parameters. Another feature of the formulation of ref. [7] is that the off-shell numbers of bosonic and fermionic degrees of freedom are not equal.

In this paper, we shall adopt a uniform group-theoretical basis for the study of all the superparticle cases. We shall identify the commuting spinor variable $\psi$ as a parameter of a coset space $\mathcal{G} / \mathcal{H}$, where $\mathcal{G}$ is the proper orthochronous Lorentz group $S O^{\uparrow}(1, d-1)$ and $\mathcal{H}$ is its maximal subgroup. This space has dimension $d-2$, it is compact and corresponds to the sphere $S^{d-2}$ (which is known as the celestial sphere [8] in the $d=4$ case). The extension of the ordinary superspace coordinates $x, \theta$ by this sphere is very reminiscent of the harmonic superspace formalism [9] in which commuting isospinor coordinates taking values in the sphere $S^{2}$ are introduced in order to organise infinite sets of auxiliary or gauge fields via harmonic expansions. In particular, the sphere $S^{d-2}$ may be viewed as the manifold of all possible choices of light-cone frames, allowing one to have the benefits of a light-cone gauge without losing Lorentz covariance, much in the same way as in the harmonic superspace formalism, where the sphere $S^{2}$ may be viewed as the manifold of all possible choices of complex structures in $N=2, d=4$ supersymmetry.

The above group-theoretical basis allows for a consistent understanding of the superparticle theories for $d=3,4 \& 6$ constructed in $[3,5,6]$. It explains some peculiarities of ref. [7] that arise as a result of a gauge fixing. It also establishes relations to the various approaches to an interpretation of the $d=10$ supersymmetric Yang-Mills and supergravity constraints as integrability conditions [10]-[13]. 
Another motivation of the present paper is to improve on previous approaches to lightcone harmonic superspace, which were based on the coset space $S O(1, d-1) /[S O(d-2) \times$ $S O(1,1)][14,15]$. The latter has dimension $2(d-2)$ and it is noncompact. There is an essential difference between the compact and noncompact coset spaces of the Lorentz group: square-integrable functions on noncompact coset spaces contain in their decomposition only infinite-dimensional representations of the Lorentz group, while the analogous functions on compact spaces may contain both finite and infinite-dimensional representations (see, for example [16]). Since ordinary physics is based on finite-dimensional representations, compact coset spaces are preferred as candidates for light-cone harmonic superspace.

The present paper is organized as follows. First, we examine a new action for the ordinary massless particle, which contains a null-vector as a dynamical variable, and establish its group-theoretical meaning as a parameter of the smallest possible coset space of the Lorentz group $S O^{\uparrow}(1, d-1)$. This coset space can be constructed either in the vector or in the spinor representation of $S O^{\uparrow}(1, d-1)$. The latter is the relevant one for the case of the superparticle. In sections 3,4 and 5 we systematically consider superparticles in $d=3,4$, and 6 from this point of view. Section 6 is devoted to the $d=10$ case. In section 7 we summarize our results and formulate some open problems; we also give an appendix devoted to the worldline superconformal groups.

\section{The bosonic particle and $S^{d-2}$}

We begin with a discussion of the ordinary bosonic relativistic particle in $d$ dimensions. Following [3], we write the action for the particle as

$$
I=\int d \tau p_{m}(\tau)\left[\dot{x}^{m}(\tau)-v^{m}(\tau)\right]
$$

where the vector $v^{m}$ is constrained to be lightlike,

$$
v^{m} v_{m}=0
$$

and must also satisfy the further covariant condition (with respect to the orthochronous subgroup $S O^{\uparrow}(1, d-1)$ of the Lorentz group)

$$
v^{0}>0
$$

Note that this inequality is necessitated by the following physical requirement: at any point of the worldline, $\tau$ can be interpreted as a time parameter; that is $\dot{x}^{m}(\tau)>0$. Hence the vector $v^{m}$ must be strictly nonvanishing. 
The action (2.1) is invariant under arbitrary worldline reparametrisations, $\tau \rightarrow \tau^{\prime}(\tau)$ which preserve the worldline orientation, i.e., they are monotonic, $d \tau^{\prime} / d \tau>0$. Note from (2.1) that $v^{m}$ must transform as a worldline density under reparametrisations

$$
v^{\prime m}\left(\tau^{\prime}\right)=\frac{d \tau}{d \tau^{\prime}} v^{m}(\tau)
$$

In order to relate (2.1) to the more familiar form of the massless particle action, one first incorporates the constraint (2.2) into the action with a Lagrange multiplier $e^{-1}$,

$$
I=\int d \tau\left(p_{m}(\tau)\left[\dot{x}^{m}(\tau)-v^{m}(\tau)\right]+\frac{1}{2} e^{-1} v^{m} v_{m}\right)
$$

and then varies with respect to $v^{m}$ to find $v_{m}=e p_{m}$, which may be substituted back into (2.5) to obtain the familiar massless particle action

$$
I=\int d \tau\left[p_{m} \dot{x}^{m}-\frac{1}{2} e p^{2}\right]
$$

An interesting feature of the action (2.1) (as compared to (2.6)) is that it is covariant under general coordinate transformations without using the einbein field $e(\tau)$ and thus it is reminiscent of Chern-Simons type actions.

The vector $v^{m}$ subject to the constraints $(2.2,2.3)$ and considered modulo the $\tau$ reparametrisations (2.4) takes its values in a sphere $S^{d-2}$. This can be seen by breaking up $v^{m}$ into $\left(v^{0}, v^{\hat{m}}\right), \hat{m}=1,2, \ldots, d-1$, and then using the $\tau$ reparametrisations to pick the gauge $v^{0}=1$ (remembering that $v^{0}$ transforms as a density), so that $v^{\hat{m}} v^{\hat{m}}=1$. Another way to see this is to pass over to gauge-invariant coordinates $\xi^{\hat{m}}=v^{\hat{m}} / v^{0}$, for which $\xi^{\hat{m}} \xi^{\hat{m}}=1$.

A group-theoretic interpretation of the space in which the Lorentz vector $v^{m}$ takes its values is in terms of the coset space $\mathcal{G} / \mathcal{H}$,

$$
S^{d-2}=\frac{S O^{\uparrow}(1, d-1)}{\left[S O(d-2) \times S O^{\uparrow}(1,1)\right] \otimes\left\{K_{\tilde{m}}\right\}},
$$

where $\mathcal{G}=S O^{\uparrow}(1, d-1)$ is the proper orthochronous Lorentz group in $d$ dimensions, while the divisor subgroup $\mathcal{H}=\left[S O(d-2) \times S O^{\uparrow}(1,1)\right] \otimes\left\{K_{\tilde{m}}\right\}$ is its maximal proper subgroup. Here $\tilde{m}=1,2, \ldots d-2,\left\{K_{\tilde{m}}\right\}$ denotes the abelian group generated by the "conformal boosts" $K_{\tilde{m}}$ and $\otimes$ denotes a semidirect product. To clearly understand the structure of the coset (2.7) it is very useful to view the $d$-dimensional Lorentz group $S O^{\uparrow}(1, d-1)$ as the conformal group of the Euclidean $(d-2)$-dimensional space. Then the Lorentz generators $L_{m n}=-L_{n m}$, which satisfy the algebra*

$$
\left[L_{m n}, L_{k l}\right]=\eta_{m k} L_{n l}-\eta_{m l} L_{n k}-(m \leftrightarrow n),
$$

* We use the "mostly plus" convention for the Minkowski tensor: $\eta_{m n}=\operatorname{diag}(-1,1, \ldots 1)$. 
can be separated into the generators of $S O(d-2)$ rotations $L_{\tilde{m} \tilde{n}}, S O^{\uparrow}(1,1)$ scale transformations $D=L_{0, d-1},(d-2)$-dimensional translations $P_{\tilde{m}}=L_{0, \tilde{m}}-L_{\tilde{m}, d-1}$ and conformal boosts $K_{\tilde{m}}=L_{0, \tilde{m}}+L_{\tilde{m}, d-1}$. The subgroup $\mathcal{H}$ is then generated by $\left\{L_{\tilde{m} \tilde{n}}, D, K_{\tilde{m}}\right\}$.

The coset (2.7) has a number of interesting features: $i$ ) its dimension is $d-2$ and it is the smallest possible coset of the Lorentz group $\mathcal{G}$. This corresponds to the fact that the subgroup $\mathcal{H}$ is the maximal proper subgroup of $\mathcal{G}$; ii) both $\mathcal{G}$ and $\mathcal{H}$ are noncompact and at the same time the coset space is compact; iii) the coset space generators $P_{\tilde{m}}$ form a maximal lightlike abelian subgroup of $\mathcal{G}$. This means that they mutually commute and the Cartan-Killing norm of these generators, as well as of any of their linear combinations, vanishes.

In order to see why (2.7) is the space that $v^{m}$ takes its values in, one can look for the maximal subgroup of the Lorentz group $S O^{\uparrow}(1, d-1)$ that preserves a given light-like vector $v^{m}$ with $v^{0}>0$, modulo dilatations of $v^{m}$. Passing to a light-cone frame, where this vector has only the first and the last components, $v^{m}=(v, 0, \ldots, 0,-v)$, it is easy to see that the sought-for subgroup coincides with $\mathcal{H}$. For this reason, the variable $v^{m}(2.2-2.4)$ parametrizes the space of all possible light-cone frames, so that its introduction amounts to covariantising the light-cone.

It is instructive to give a global description of the coset space (2.7), starting from a matrix $v_{a}^{m}$ that is an element of $S O^{\uparrow}(1, d-1)$, so that it satisfies

$$
\begin{aligned}
\eta_{m n} v^{m}{ }_{a} v_{b}^{n} & =\eta_{a b} \\
\operatorname{det}\left(v^{m}{ }_{a}\right) & =1 \\
v_{0}^{0} & \geq 1 .
\end{aligned}
$$

As usual in nonlinear realisations, we define the group action of $\mathcal{G}=S O^{\uparrow}(1, d-1)$ by left multiplication,

$$
v^{\prime m}{ }_{a}=\Lambda_{n}^{m} v_{a}^{n},
$$

where $\Lambda^{m}{ }_{n} \in \mathcal{G}$. On the other hand, in constructing (2.7), $v^{m}{ }_{a}$ must be considered to be defined only up to multiplication on the right by arbitrary elements of the divisor group $\mathcal{H}=$ $\left[S O(d-2) \times S O^{\uparrow}(1,1)\right] \otimes\left\{K_{\tilde{m}}\right\}$

$$
v^{\prime m}{ }_{a}=v^{m} \Omega_{a}^{b}
$$

where $\Omega^{b}{ }_{a} \in \mathcal{H}$.

Now, although the left index $m$ on $v^{m}{ }_{a}$ corresponds to an irreducible (vector) representation of $S O^{\uparrow}(1, d-1)$, the right index $a$ corresponds to a representation of the divisor group that is reducible, but not completely reducible, i.e. it can be transformed into block triangular form, but not into block diagonal form. It is easiest to see this using a light-cone 
basis for the indices $a, b, \ldots$ of $\mathcal{H}$ (note that this step does not break the Lorentz group acting on the indices $m, n, \ldots)$ :

$$
v_{ \pm}^{m}=\frac{1}{\sqrt{2}}\left(v_{0}^{m} \pm v_{d-1}^{m}\right) .
$$

In this basis, the $\left[S O(d-2) \times S O^{\uparrow}(1,1)\right] \otimes\left\{K_{\tilde{m}}\right\}$ "gauge transformations" have the uppertriangular form

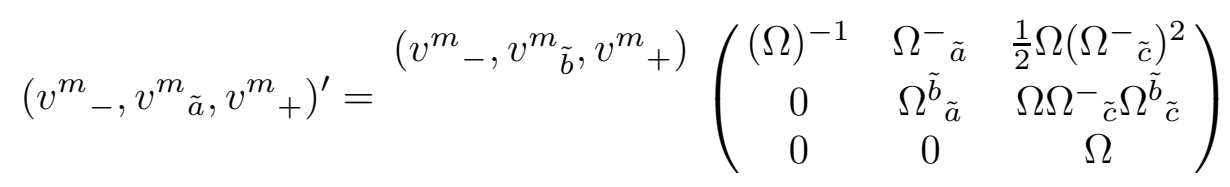

where $\Omega$ is a positive $S O^{\uparrow}(1,1)$ scale transformation parameter, $\Omega^{\tilde{b}} \in S O(d-2)$ and $\Omega^{-} \tilde{b}$ corresponds to the $\left\{K_{\tilde{m}}\right\}$ transformations.

Note that $v^{m}$ - transforms homogeneously in (2.13)

$$
v_{-}^{\prime m}=(\Omega)^{-1} v_{-}^{m}
$$

while $v^{m} \tilde{a}$ and $v^{m}+$ transform into themselves as well as into $v^{m}$ - (and hence the incomplete reducibility of the representation).

The matrix components $v^{m}, v^{m} \tilde{a}$, and $v^{m}$ + satisfy the following constraints, which are just (2.9) written in the light-cone basis (2.11):

$$
\begin{aligned}
\eta_{m n} v^{m} v_{-} v_{-} & =0 \\
\eta_{m n} v^{m}{ }_{+} v_{+}^{n} & =0 \\
\eta_{m n} v^{m}+v^{n}- & =-1 \\
\eta_{m n} v^{m}{ }_{ \pm} v_{\tilde{a}}^{n} & =0 \\
\eta_{m n} v^{m}{ }_{\tilde{a}} v_{\tilde{b}}^{n} & =\delta_{\tilde{a} \tilde{b}} \\
\operatorname{det}\left(v_{-}^{m}, v^{m}{ }_{\tilde{a}}, v^{m}\right) & =1 \\
v^{0}{ }_{-} & >0 .
\end{aligned}
$$

The condition (2.15g) follows from $\eta^{a b} v_{a}^{m} v_{b}^{n}=\eta^{m n}$ (conjugate to (2.9a)) and (2.9c).

In order to see that the manifold $(2.7)$ is $S^{d-2}$, we shall now consider the first column $v^{m}$ and note from (2.14) that owing to the local $S O^{\uparrow}(1,1)$ symmetry, this vector is defined only up to a local scale factor. At this point, we can establish the link with the particle's variable $v^{m}$, which is to be identified with $v^{m}$, while the local $S O^{\uparrow}(1,1)$ symmetry acting on it is to be identified with the $\tau$ reparametrisation symmetry (2.4). Being a column of an orthogonal matrix this vector is naturally nonvanishing. From $(2.15 \mathrm{a})$, we see that $v^{m}=v^{m}$ - is a null vector, as required by the variational principle $(2.1),(2.2)$. Finally, the time component of this vector is positive $(2.15 \mathrm{~g})$ in accordance with $(2.3)$. 
Given $v^{m}$, the remaining components $v^{m} \tilde{a}$ and $v^{m}$ are all eliminable, either by the remaining constraints $(2.15 \mathrm{~b}-\mathrm{f})$ or by the $S O(d-2) \otimes\left\{K_{\tilde{m}}\right\}$ gauge transformations. The best way to see how this may be done is to use the $S O^{\uparrow}(1, d-1)$ covariance of $(2.15)$ and transform the vector $v^{m}$ - to a special form such as $(1,0, \ldots, 0,-1)$, in which $(2.15 \mathrm{a}-\mathrm{f})$ take an especially simple form that allows one to see that $v^{0}+v^{d-1}+, v^{0} \tilde{a}$ and $v^{d-1} \tilde{a}$ can all be solved for in terms of $v^{\tilde{m}}+$ and $v^{\tilde{m}} \tilde{a} \in S O(d-2)$. These may then be gauged away by the $\left\{K_{\tilde{m}}\right\}$ and the $S O(d-2)$ transformations, with parameters $\Omega^{-} \tilde{a}$ and $\Omega^{\tilde{b}} \tilde{a}(2.13)$ respectively.

Thus we have shown that the constraints (2.15) for the space (2.7), defined in terms of the $v^{m}{ }_{a}$ modulo the divisor group $\left[S O(d-2) \times S O^{\uparrow}(1,1)\right] \otimes\left\{K_{\tilde{m}}\right\}$, give the same manifold as that defined by a vector $v^{m}$ that is required to satisfy $(2.2,2.3)$ and defined modulo reparametrisations (2.4). As we have showed earlier, this space is the sphere $S^{d-2}$.

The Lorentz-covariant parametrisation of the sphere $S^{d-2}$ by the matrix $v^{m}{ }_{a}$ differs from the familiar parametrisation that underlies ordinary spherical harmonics based upon the coset $S O(d-1) / S O(d-2)=S^{d-2}$. We may relate these two by focusing on the vector harmonics. After elimination of $v^{m} \tilde{a}$ and $v^{m}+$ by the constraints (2.15) and use of the $S O(d-2)$ and $\left\{K^{\tilde{m}}\right\}$ gauge freedoms, the remaining light-like $v^{m}=v^{m}$ - may be written as $v^{m}=\left(R, R v^{\hat{m}}\right), \hat{m}=1, \ldots, d-1$, where $v^{\hat{m}} v^{\hat{m}}=1$. The $v^{\hat{m}}$ can then be identified with the usual vector spherical harmonics. Higher spherical harmonics can then be obtained as symmetrised traceless products of the $v^{\hat{m}}$. The problem of constructing general Lorentz covariant functions in terms of the $v^{m}{ }_{a}$ is the same as that of constructing conformally covariant functions on $S^{d-2}$. An essential feature of this construction is the use of functions with a homogeneous scaling behaviour under the $S O^{\uparrow}(1,1)$ symmetry. The construction of general conformally covariant functions in this way has been treated in ref. [16]. In the present paper, we shall not discuss further the construction of general Lorentz-covariant functions necessary for field-theoretical applications, but shall restrict our attention to the invariance properties of particle actions such as (2.1).

We end this section with the following comment. One may relax the positivity conditions on $v^{0}(2.3)$ and $d \tau^{\prime} / d \tau$, by requiring instead that they be nonvanishing. This corresponds to an equivalent representation for the coset space (2.7)

$$
S^{d-2}=\frac{S O(1, d-1)}{[S O(d-2) \times S O(1,1)] \otimes\left\{K^{\tilde{m}}\right\}} .
$$

However, the above positivity conditions naturally appear in the superparticle theory, to which we now turn.

\section{The superparticle in $d=2+1$}

For the superparticle, the coset (2.7) is still the space that the $v^{m}$ take their values in, but in order to accommodate the superparticle's spinor variable, we now make use of the 
fundamental spinor representation of the Lorentz group. In other words, we will consider the smallest coset space of the $\operatorname{Spin}(1, d-1)$ group, which is the covering group of $S O^{\uparrow}(1, d-1)$. Since the spinor representations grow exponentially in $d$ compared to the vector representation used above, there will now be a dimensional dependence in our treatment. We begin in $d=2+1$, where $\operatorname{Spin}(1,2)=S L(2, \mathbb{R})$ and the coset $(2.7)$ is represented starting from a real unimodular $2 \times 2$ matrix $e^{\mu}{ }_{\alpha} \in S L(2, \mathbb{R})$, where the greek indices denote spinor representations and run over the values 1,2 . As in the bosonic case above, the $S L(2, \mathbb{R})$ transformations act by left multiplication,

$$
e^{\prime \mu}{ }_{\alpha}=\Lambda_{\nu}^{\mu} e_{\alpha}^{\nu}
$$

where $\Lambda \in S L(2, \mathbb{R})$, so $\operatorname{det} \Lambda=1$. The maximal subgroup $\mathcal{H}_{B}$ of $S L(2, \mathbb{R})$ (which in this case is known as the Borel subgroup) is given by the upper-triangular matrices

$$
h=\left(\begin{array}{cc}
a & b \\
0 & a^{-1}
\end{array}\right), \quad a \neq 0 \quad a, b \in \mathbb{R} .
$$

(Strictly speaking, this $\mathcal{H}_{B}$ differs from $S O^{\uparrow}(1,1) \otimes\{K\}$ by a $Z_{2}$ factor, corresponding to the possibility of both positive and negative values for $a$ in (3.2). We shall return to the issue of the double coverings below.) The right-hand index $\alpha$ corresponds to a reducible representation of $\mathcal{H}_{B}$, with gauge transformations

$$
\begin{aligned}
\psi^{\prime \mu} & =a \psi^{\mu} \\
\phi^{\prime \mu} & =a^{-1} \phi^{\mu}+b \psi^{\mu}
\end{aligned}
$$

where $\psi^{\mu}=e^{\mu}{ }_{1}, \phi^{\mu}=e^{\mu}{ }_{2}$.

The coset space (2.7) in this $d=2+1$ case is $S^{1}$. Since this one-dimensional space has fewer components than are contained in the $e^{\mu}{ }_{\alpha}$, the number of independent components of this matrix must be reduced to just one. This happens as a result of two particular features of our construction. The first is that the matrix $e^{\mu}{ }_{\alpha}$ is required to be an element of $S L(2, \mathbb{R})$, and so there is a constraint. In terms of the $(\psi, \phi)$ variables introduced above, this is, from $\operatorname{det}\left(e_{\alpha}^{\mu}\right)=1$,

$$
\psi^{1} \phi^{2}-\psi^{2} \phi^{1}=1
$$

We also have the restriction that $\psi^{\mu} \neq 0$, since this would otherwise be inconsistent with $\operatorname{det}\left(e_{\alpha}^{\mu}\right)=1$. This means that either $\psi^{1} \neq 0$ or $\psi^{2} \neq 0$. These cases define for us the two charts needed to cover $S^{1}$. Consider to be specific the first case. In this case, we may solve the constraint (2.4) for $\phi^{2}$ :

$$
\phi^{2}=\frac{1}{\psi^{1}}-\psi^{2} \frac{\phi^{1}}{\psi^{1}}
$$


We now have three remaining degrees of freedom. Two of these may be removed using the second particular feature of our construction, namely the $\mathcal{H}_{B}$ gauge transformations

$$
\begin{aligned}
\phi^{\prime 1} & =a^{-1} \phi^{1}+b \psi^{1} \\
\psi^{\prime \mu} & =a \psi^{\mu}
\end{aligned}
$$

Thus, we may pick, e.g., the gauge $\phi^{1}=0$, and take $x=\psi^{2} / \psi^{1}$ as a gauge invariant coordinate in this chart. In the other chart, where $\psi^{2} \neq 0$, one proceeds analogously but dividing by $\psi^{2}$, and ending up with the remaining coordinate $y=\psi^{1} / \psi^{2}$. In the overlapping region of these charts $x \neq 0, y \neq 0$ and $x y=1$, so $x$ and $y$ can be viewed as stereographic coordinates on the circle, arising from projection from the north and south poles onto the equatorial line.

In order to clarify the discussion of the higher-dimensional cases to follow, we now consider in more detail the relation between the spinor representation construction of (3.1$3.4)$ and the vector representation construction based on $S O^{\uparrow}(1,2)$ (see $(2.9),(2.13)$ ). The spinor and vector representation matrices are related by

$$
e^{\mu}{ }_{\alpha} e^{\nu}{ }_{\beta} \sigma_{\mu \nu}^{m}=v^{m}{ }_{a} \sigma_{\alpha \beta}^{a},
$$

where

$$
\sigma_{\mu \nu}^{m}=\left(\begin{array}{ll}
1 & 0 \\
0 & 1
\end{array}\right), \quad\left(\begin{array}{ll}
0 & 1 \\
1 & 0
\end{array}\right), \quad\left(\begin{array}{cc}
-1 & 0 \\
0 & 1
\end{array}\right), \quad m=0,1,2 .
$$

Given $e^{\mu}{ }_{\alpha}$, we find $v^{m}{ }_{a}$ as

$$
v^{m}{ }_{a}=-\frac{1}{2} e^{\mu}{ }_{\alpha} e^{\nu}{ }_{\beta} \sigma_{\mu \nu}^{m} \tilde{\sigma}_{a}^{\alpha \beta}
$$

where $\tilde{\sigma}^{m \mu \nu}=\epsilon^{\mu \mu^{\prime}} \epsilon^{\nu \nu^{\prime}} \sigma_{\mu^{\prime} \nu^{\prime}}^{m}$, and $\operatorname{tr}\left(\sigma^{m} \tilde{\sigma}^{n}\right)=-2 \eta^{m n}$. Note that $v_{0}^{0}$ is strictly positive, $v_{0}^{0}=\frac{1}{2}\left(\left(e^{1}{ }_{1}\right)^{2}+\left(e^{1}{ }_{2}\right)^{2}+\left(e^{2}{ }_{1}\right)^{2}+\left(e^{2}{ }_{2}\right)^{2}\right)>0$.

One can easily check that the unimodularity constraint on $e^{\mu}{ }_{\alpha}$ is equivalent to the requirement that $v^{m}{ }_{a} \in S O^{\uparrow}(1,2)$ and that the spinorial matrix $e^{\mu}{ }_{\alpha}$ is defined by the vectorial matrix $v^{m}$ up to a sign, in accordance with

$$
\frac{S L(2, \mathbb{R})}{Z_{2}}=\frac{\operatorname{Spin}(1,2)}{Z_{2}}=S O^{\uparrow}(1,2)
$$

Although we have already shown that our coset space (2.7) in the $d=2+1$ case is the circle $S^{1}$, it is instructive to use the relation of the spinor representation to the vector representation (3.8) in order to see once again that the manifold is $S^{1}$. In the $d=2+1$ case, the transverse $\tilde{a}$ index in (2.7) can take only the value $\tilde{a}=1$. Thus, we need to construct 
the $v^{m}-v^{m}$ and $v^{m}+$ vectors from the $\psi^{\mu}$ and $\phi^{\mu}$ spinors. Rewriting the lower $a$ index in the light-cone basis (2.11) we get

$$
\begin{aligned}
v_{-}^{m} & =\frac{1}{\sqrt{2}} \sigma_{\mu \nu}^{m} \psi^{\mu} \psi^{\nu} \\
v^{m}{ }_{1} & =\sigma_{\mu \nu}^{m} \psi^{\mu} \phi^{\nu} \\
v_{+}^{m} & =\frac{1}{\sqrt{2}} \sigma_{\mu \nu}^{m} \phi^{\mu} \phi^{\nu} .
\end{aligned}
$$

It is important to emphasize that in such a representation $v^{m}$ - is automatically a null vector: it is expressed in terms of a two-component spinor $\psi^{\mu}$, and it is impossible to construct a Lorentz scalar from $\psi^{\mu}$ alone. From the spinor representation gauge transformations (3.3), one recovers the vector representation gauge transformations (2.13). Then, by our previous discussion for the vector representation, we know that the manifold $S L(2, R) / \mathcal{H}_{B}$ is a circle. Since $\mathcal{H}_{B} / Z_{2}=\mathcal{H}=S O^{\uparrow}(1,1) \otimes\{K\}$ and $S L(2, \mathbb{R}) / Z_{2}=S O^{\uparrow}(1,2)$, this spinor realisation of the coset describes the same manifold $S^{1}$ as the vector construction (2.7) (since the $Z_{2}$ factors "cancel out").

The main benefit of the spinor formulation is that it allows for a natural interpretation of the $\kappa$-symmetry of the superparticle as a local worldline supersymmetry. In particular, this permits the $\kappa$ symmetry to be reformulated with an algebra that closes without the use of equations of motion. Staying for the moment with the $d=2+1$ case, and following [3], we consider the target space coordinates $x^{m}(\tau)$ and $\theta^{\mu}(\tau)$ as the lowest components of superfields $X^{m}, \Theta^{\mu}$ on an $N=1$ worldline superspace with coordinates $(\tau, \eta)$. As usual, we may expand these superfields into component fields using a superspace covariant derivative

$$
D=\partial_{\eta}+\mathrm{i} \eta \partial_{\tau}
$$

which satisfies

$$
D^{2}=\mathrm{i} \partial_{\tau}
$$

For an unrestricted local worldline supersymmetry, it would be necessary to include a "super einbein" in the construction of $D$, but this einbein can always be gauged away, leaving $D$ in the form (3.13). The residual covariance of $D$ is then restricted to transformations of the form

$$
\begin{aligned}
& \delta \tau=\Lambda-\frac{1}{2} \eta D \Lambda=\lambda(\tau)+\mathrm{i} \eta \epsilon(\tau) \\
& \delta \eta=-\frac{\mathrm{i}}{2} D \Lambda=\epsilon(\tau)+\frac{1}{2} \eta \dot{\lambda}(\tau),
\end{aligned}
$$

where the components of the superfield parameter $\Lambda(\tau, \eta)=\lambda(\tau)+2 \mathrm{i} \eta \epsilon(\tau)$ correspond to ordinary worldline diffeomorphisms and local worldline supersymmetry. By the residual covariance of (3.13) under (3.15), we mean that $D$ transforms into a factor times itself, i.e.

$$
\delta D=-(D \delta \eta) D
$$


Eq. (3.16) defines a superconformal transformation of the super worldline (see appendix). Under the local transformations $(3.15), \Theta(\tau, \eta)$ transforms as a scalar. The superfield $\Theta(\tau, \eta)$ can be expanded into component fields as

$$
\theta^{\mu}(\tau)=\left.\Theta^{\mu}\right|_{\eta=0}, \quad \psi^{\mu}(\tau)=\left.D \Theta^{\mu}\right|_{\eta=0}
$$

As a consequence, the worldline supersymmetry transformations of $\theta(\tau)$ are

$$
\delta \theta^{\mu}(\tau)=-\epsilon(\tau) \psi^{\mu}(\tau)
$$

This transformation can be related to the familiar $\kappa$-symmetry if we choose the parameter $\epsilon$ to be field-dependent,

$$
\epsilon=\kappa_{\nu} \psi^{\nu}
$$

and recall, from (3.12a) with the identification $v^{\mu \nu}=\tilde{\sigma}_{m}^{\mu \nu} v^{m}=v^{(\mu \nu)}$, that $v^{\mu \nu}=\frac{1}{\sqrt{2}} \psi^{\mu} \psi^{\nu}$ so that the right hand side of (3.18) contains a light-like vector that is proportional on shell (c.f. eq. (2.3)) to $p^{\mu \nu}=\sigma_{m}^{\mu \nu} p^{m}$. This reproduces the standard form of the $\kappa$-symmetry transformation

$$
\delta \theta^{\mu}(\tau) \sim p^{\mu \nu} \kappa_{\nu}
$$

Note that in making the field-dependent substitution (3.19), one introduces a twocomponent spinor anticommuting parameter $\kappa_{\mu}$ which contains twice as many degrees of freedom as $\epsilon$. Thus it is not surprising that $\kappa_{\mu}$ is defined modulo its own "pre-gauge" transformations

$$
\delta \kappa_{\mu}=\xi(\tau) \psi_{\mu}
$$

If one were to insist on using only objects with the standard spin-statistics correlation (anticommuting half-integer spins and commuting integer ones), it would be necessary to put $\xi(\tau)=\rho^{\nu}(\tau) \psi_{\nu}$ so that $\delta \kappa_{\mu} \sim p_{\mu \nu} \rho^{\nu}$; this would start an infinite sequence of pregauge transformations.

The superparticle action in the $d=2+1$ case has both the restricted local worldline supersymmetry (2.15) and also a rigid target-space superconformal symmetry [3]:

$$
I_{d=2+1}=-\mathrm{i} \int d \tau d \eta P_{\mu \nu}\left(D X^{\mu \nu}-\mathrm{i} D \Theta^{(\mu} \Theta^{\nu)}\right)
$$

The component expansions of $X^{\mu \nu}$ and $P^{\mu \nu}$ are obtained as above by expanding using $D$,

$$
\begin{aligned}
x^{\mu \nu}(\tau)=\sigma_{m}^{\mu \nu} x^{m}(\tau) & =\left.X^{\mu \nu}\right|_{\eta=0} \\
p^{\mu \nu}(\tau) & =\left.P^{\mu \nu}\right|_{\eta=0}
\end{aligned}
$$


while the higher components of both of these superfields are auxiliary. Upon elimination of the auxiliary fields in (3.22), one obtains

$$
I=\int d \tau p_{\mu \nu}\left(\dot{x}^{\mu \nu}-\psi^{\mu} \psi^{\nu}-\mathrm{i} \dot{\theta}^{(\mu} \theta^{\nu)}\right)
$$

Since one has eliminated auxiliary fields to obtain this form of the action, the worldline supersymmetry transformations form a closed algebra at this stage only modulo the equations of motion. In order to recover the conventional superparticle action, one should vary (3.24) with respect to $\psi^{\mu}$, obtaining

$$
p_{\mu \nu} \psi^{\mu}=0
$$

which may in turn be solved by

$$
p_{\mu \nu}=e^{-1} \psi_{\mu} \psi_{\nu}
$$

since $\psi_{\mu}$ is nonvanishing. Substituting for $\psi^{\mu}$ then reproduces the usual form of the action

$$
\int d \tau\left[p_{\mu \nu}\left(\dot{x}^{\mu \nu}-\mathrm{i} \dot{\theta}^{(\mu} \theta^{\nu)}\right)-\frac{1}{2} e p_{\mu \nu} p^{\mu \nu}\right]
$$

Subject to the equations of motion following from (3.22), there is a rigid part of the $d=1$ worldline supersymmetry (3.15) that may be viewed as a projection of the target spacetime supersymmetry. This rigid worldline supersymmetry may also be defined as the transformations under which the superspace derivative $D(3.13)$ is invariant. In the projection, $D$ may be viewed as a "spinorial pull-back" of the target superspace derivative $D_{\mu}$. This projection will help us to clarify why the worldline supersymmetry has half the number of generators of the spacetime supersymmetry, which in this case yields an unextended $N=1$ worldline supersymmetry.

The target superspace derivative is

$$
D_{\mu}=\frac{\partial}{\partial \Theta^{\mu}}+\mathrm{i} \Theta^{\nu} \frac{\partial}{\partial X^{\mu \nu}}
$$

and satisfies the algebra

$$
\begin{aligned}
& \left\{D_{\mu}, D_{\nu}\right\}=2 \mathrm{i} D_{\mu \nu} \\
& {\left[D_{\mu \nu}, D_{\rho}\right]=0}
\end{aligned}
$$

where $D_{\mu \nu}=\partial /\left(\partial X^{\mu \nu}\right)$. Now in order to make an identification between the worldline supersymmetry and a projected version of this algebra, we will want to use the chain rule together with a specific solution of the superparticle equations of motion, taken in an appropriate gauge for the local worldline transformations (3.15). The equations of motion 
following from (3.22) expand into component fields to give

$$
\begin{aligned}
\dot{p}_{\mu \nu} & =0 \\
\dot{x}^{\mu \nu} & =\psi^{\mu} \psi^{\nu}+\mathrm{i} \dot{\theta}^{(\mu} \theta^{\nu)} \\
p_{\mu \nu} \psi^{\nu} & =0 \\
p_{\mu \nu} \dot{\theta}^{\mu} & =0 .
\end{aligned}
$$

The solution to (3.30c) was given in (3.26); at this point it is convenient to fix the $\tau$ reparametrisation gauge in (3.15) by setting

$$
\dot{e}=0
$$

(3.30a) then implies

$$
\psi^{\mu}=\psi_{(0)}^{\mu}=\text { constant. }
$$

Eq. (2.30d) then implies

$$
\dot{\theta}^{\mu}=\psi_{(0)}^{\mu} \zeta(\tau),
$$

where $\zeta(\tau)$ is an arbitrary Grassmann function, but one may then use the worldline supersymmetry freedom $\epsilon(\tau)$ in (3.18) to set

$$
\xi(\tau)=0,
$$

So

$$
\theta^{\mu}(\tau)=\theta_{(0)}^{\mu}=\text { constant. }
$$

Finally, we may solve the $x^{\mu \nu}$ equation (3.30b) by

$$
x^{\mu \nu}(\tau)=x_{(0)}^{\mu \nu}+\psi_{(0)}^{\mu} \psi_{(0)}^{\nu} \tau .
$$

The covariant derivatives on the target superspace can then be projected onto the worldline using $\psi_{(0)}^{\mu}$ :

$$
\begin{aligned}
D & =\psi_{(0)}^{\mu} D_{\mu} \\
\partial_{\tau} & =\psi_{(0)}^{\mu} \psi_{(0)}^{\nu} D_{\mu \nu},
\end{aligned}
$$

with the resulting algebra

$$
\begin{aligned}
D^{2} & =\mathrm{i} \partial_{\tau} \\
{\left[D, \partial_{\tau}\right] } & =0 .
\end{aligned}
$$

The differential operators $D$ and $\partial_{\tau}$ are understood to act on functions of $(X(\tau, \eta), \Theta(\tau, \eta))$, where the worldline component fields of $X(\tau, \eta)$ and $\Theta(\tau, \eta)$ are given in $(3.32,3.35,3.36)$. 
The gauge choices (3.31) and (3.34) leave unfixed a rigid $N=1$ worldline supersymmetry, under which the worldline superspace derivative $D(3.37)$ is invariant.

In addition to the projected supersymmetry transformations, in the $d=2+1$ case there is a scale transformation automorphism symmetry of the $D, \partial_{\tau}$ algebra (3.16) that can be identified with the $S O^{\uparrow}(1,1)$ part of the Borel subgroup $\mathcal{H}_{B}$. In fact, one may say that the whole of $\mathcal{H}_{B}$ acts on the algebra (3.14), but that the derivatives $D$ and $\partial_{\tau}$ are inert under the $K_{\tilde{m}}$ transformations. This is easy to see using the projection relations $(3.37,3.38)$, since $\psi_{(0)}^{\mu}$ is invariant under $K_{\tilde{m}}$ as is evident from (3.3).

The appearance of $N=1$ worldline supersymmetry in the projected algebra of the operators $(3.37,3.38)$ is illustrative of the general situation, in which the worldline supersymmetry has half the number of components of the target spacetime supersymmetry. This is well-known from light-cone considerations. The analysis of the present paper amounts to a Lorentz covariantisation of the light-cone; the halving of the number of supersymmetries on the worldline carries over directly. This can be seen already from a group-theoretical standpoint, for of the two spinors potentially available to make projections of $D_{\mu}$ only $\psi^{\mu}$ transforms homogeneously under $\mathcal{H}_{B}$ transformations: as one can see again from $(3.3), \phi^{\mu}$ transforms into $\psi^{\mu}$ under the $K_{\tilde{m}}$ transformation. As a result, only $\psi^{\mu}$ may be used to make a $K_{\tilde{m}}$-invariant projected worldline derivative (3.37) so that there is only one type of " $\partial_{\tau}$ " derivative occurring in the closure of the $D$ algebra, that given in (3.38). If we had tried to start from $\phi^{\mu} D_{\mu}$ (or to include it into the algebra together with $\psi^{\mu} D_{\mu}$ ), closure of the "worldline" $D$ algebra together with the $\mathcal{H}_{B}$ transformations would then lead to three independent bosonic derivatives, $\psi^{\mu} \psi^{\nu} D_{\mu \nu}, \psi^{\mu} \phi^{\nu} D_{\mu \nu}$ and $\phi^{\mu} \phi^{\nu} D_{\mu \nu}$. In other words, there would be no projection onto the worldline, since effectively all three of the target spacetime derivatives would appear.

4. $d=3+1$

In the case of the superparticle in $d=3+1$ dimensions, as is well known, the $\operatorname{Spin}(3,1)$ covering group of the Lorentz group is isomorphic to $S L(2, \mathbb{C})$. Accordingly, in order to obtain a spinorial realisation of the coset (2.7) for this case, we begin with a unimodular $2 \times 2$ complex matrix $e^{\mu}{ }_{\alpha} \in S L(2, \mathbb{C})$, thus satisfying again the constraint

$$
\operatorname{det}\left(e^{\mu}{ }_{\alpha}\right)=1
$$

The $S L(2, \mathbb{C})$ Lorentz transformations act on this matrix from the left,

$$
e^{\prime \mu}{ }_{\alpha}=\Lambda_{\nu}^{\mu} e_{\alpha}^{\nu}
$$

where $\Lambda \in S L(2, \mathbb{C})$. The maximal subgroup $\mathcal{H}_{B}$ of $S L(2, \mathbb{C})$ consists of the upper triangular complex matrices

$$
h=\left(\begin{array}{cc}
a & b \\
0 & a^{-1}
\end{array}\right), \quad a \neq 0 \quad a, b \in \mathbb{C} .
$$


Under the group $\mathcal{H}_{B}$ acting by right multiplication, the $\alpha$ index on $e^{\mu}{ }_{\alpha}$ becomes reducible but not fully reducible: for

$$
e^{\mu}{ }_{\alpha}=\left(\psi^{\mu}, \phi^{\mu}\right) \in S L(2, \mathbb{C}) \quad \alpha=1,2
$$

the $\mathcal{H}_{B}$ transformations are

$$
\begin{aligned}
\psi^{\prime \mu} & =a \psi^{\mu} \\
\phi^{\prime \mu} & =a^{-1} \phi^{\mu}+b \psi^{\mu} .
\end{aligned}
$$

The coset space $S L(2, \mathbb{C}) / \mathcal{H}_{B}$ in this $d=3+1$ case is just the sphere $S^{2}$. One way to see this is by studying the charts corresponding to the different solutions of (4.1) and their corresponding gauge choices, as in (3.4-3.7). Another way is to relate, in analogy with (3.8), the above spinor representation to the vector one via the $\sigma$-matrix invariance equation

$$
e_{\alpha}^{\mu} e_{\dot{\alpha}}^{\dot{\mu}} \sigma_{\mu \dot{\mu}}^{m}=v^{m}{ }_{a} \sigma_{\alpha \dot{\alpha}}^{a},
$$

where $e_{\dot{\alpha}}^{\dot{\mu}}=\overline{\left(e^{\mu} \alpha\right)}$ and $\sigma_{\mu \dot{\mu}}^{m}$ are the usual Van der Waerden matrices. From eq. (4.6) one finds the vector representation matrix

$$
v^{m}{ }_{a}=-\frac{1}{2} e^{\mu}{ }_{\alpha} e^{\dot{\mu}}{ }_{\dot{\alpha}} \sigma_{\mu \dot{\mu}}^{m} \tilde{\sigma}_{a}^{\alpha \dot{\alpha}} .
$$

which belongs to $S O^{\uparrow}(1,3)$ provided $e^{\mu}{ }_{\alpha} \in S L(2, \mathbb{C})$. Note that the sign of $v^{0}{ }_{0}$ is automatically positive since $v^{0}{ }_{0}$ is given by a positive quadratic form in the components of $e^{\mu}{ }_{\alpha}$ and $e_{\dot{\alpha}}^{\dot{\mu}}$. Furthermore, one can follow the pattern of our $d=2+1$ discusion in section 3 by noting that the $\mathcal{H}_{B}$ transformations on $e^{\mu}{ }_{\alpha}$ imply via (4.7) the $\mathcal{H}$ transformations on $v^{m}{ }_{a}$. Note that the expression for the light-like vector $v^{m}$ is now

$$
v^{m}=\frac{1}{\sqrt{2}} \sigma_{\mu \dot{\mu}}^{m} \psi^{\mu} \bar{\psi}^{\dot{\mu}} .
$$

With the spinorial coset parameter $\psi^{\mu}$, we can project the $d=3+1$ spacetime covariant derivative algebra

$$
\begin{aligned}
& \left\{D_{\mu}, D_{\nu}\right\}=0 \\
& \left\{D_{\mu}, \bar{D}_{\dot{\nu}}\right\}=2 \mathrm{i} \sigma_{\mu \dot{\nu}}^{m} \partial_{m}
\end{aligned}
$$

onto the worldline, following the pattern of our $d=2+1$ discussion $(3.37,3.38)$ (but with $\psi^{\mu}$ now complex). The pulled-back derivatives are:

$$
\begin{aligned}
D & =\psi^{\mu} D_{\mu} \\
\bar{D} & =\bar{\psi}^{\dot{\mu}} \bar{D}_{\dot{\mu}} . \\
\partial_{\tau} & =\psi^{\mu} \bar{\psi}^{\dot{\nu}} \sigma_{\mu \dot{\nu}}^{m} \partial_{m} .
\end{aligned}
$$


The resulting algebra corresponds to a rigid $N=2$ worldline supersymmetry:

$$
\begin{aligned}
\{D, \bar{D}\} & =2 \mathrm{i} \partial_{\tau} \\
D^{2} & =\bar{D}^{2}=0 .
\end{aligned}
$$

The worldline algebra (4.11) possesses an automorphism algebra that, like the $d=2+1$ case of section 3, contains scale transformations that may be identified with the $S O^{\uparrow}(1,1)$ part of $\mathcal{H}_{B}$. In the $d=3+1$ case, however, there is also a $U(1)$ automorphism that acts on $(D, \bar{D})$; this may be identified with the $S O(2)$ part of $\mathcal{H}_{B}$. As in the $d=2+1$ case, the $K^{\tilde{m}}$ transformations act trivially on $\left(D, \bar{D}, \partial_{\tau}\right)$, as one can see from (4.5) and (4.10).

The rigid $N=2$ worldline supersymmetry may be generalised directly to the local $N=2, d=1$ superconformal one (see appendix). The simplest way to describe the latter is not in terms of a real superspace $\mathbb{R}^{(1 \mid 2)}:(\tau, \eta, \bar{\eta})$, but in terms of a chiral worldline superspace $[6] \mathbb{C}^{(1 \mid 1)}:\left(\tau_{L}, \eta\right)$, where

$$
\tau_{L}=\tau+\mathrm{i} \eta \bar{\eta}
$$

The restricted worldline supersymmetry then consists of the transformations

$$
\begin{array}{r}
\delta \tau_{L}=\Lambda-\bar{\eta} \bar{D} \Lambda=\lambda\left(\tau_{L}\right)+2 \mathrm{i} \eta \bar{\epsilon}\left(\tau_{L}\right), \\
\delta \eta=-\frac{\mathrm{i}}{2} \bar{D} \Lambda=\epsilon\left(\tau_{L}\right)+\eta\left(\frac{1}{2} \dot{\lambda}+\mathrm{i} \rho\left(\tau_{L}\right) .\right.
\end{array}
$$

The parameters $\lambda, \epsilon$ and $\rho$ correspond respectively to worldline reparametrisations, local supersymmetry and local $U(1)$ transformations.

As in section 3, we may now relate the superspace transformations (4.13) to $\kappa$-symmetry transformations. Here, the worldline superfields containing the component variables $x^{m}$ and $\theta^{\mu}$ are now chiral superfields $X_{L}^{m}\left(\tau_{L}, \eta\right)$ and $\Theta^{\mu}\left(\tau_{L}, \eta\right)$. Following the pattern of (3.17), one identifies the $\eta=0$ component of $\Theta^{\mu}\left(\tau_{L}, \eta\right)$ with $\theta^{\mu}(\tau)$; the next higher component is then identified with the $d=3+1$ complex commuting spinor variable $\psi^{\mu}$ of (4.4). The worldline supersymmetry transformation is then $\delta \theta^{\mu}(\tau)=-\epsilon(\tau) \psi^{\mu}(\tau)$ (like (3.18), except that $\theta, \epsilon$ and $\psi^{\mu}$ are now all complex). One may then choose the parameter $\epsilon(\tau)$ to take the field-dependent value $\epsilon(\tau)=\bar{\kappa}_{\dot{\nu}}(\tau) \bar{\psi}^{\dot{\nu}}(\tau)$ as in (2.19). With this parameter, the variation of $\theta^{\mu}(\tau)$ takes the form $\delta \theta^{\mu}=\psi^{\mu} \bar{\psi}^{\dot{\nu}} \bar{\kappa}_{\dot{\nu}}$. On shell, $\psi^{\mu} \bar{\psi}^{\dot{\nu}}$ is proportional to $p^{\mu \dot{\nu}}$, so that using the equations of motion these transformations give rise to the standard form of a $\kappa$-transformation.

The equation of motion needed to make the identification of $\kappa$-symmetry with the local worldline supersymmetry,

$$
p_{m} \sigma_{\mu \dot{\nu}}^{m} \bar{\psi}^{\dot{\nu}}=0
$$


follows directly from the superfield form of the $d=3+1$ superparticle equations of motion for $X_{L}^{m}\left(\tau_{L}, \eta\right)$ and $\Theta^{\mu}\left(\tau_{L}, \eta\right)$ :

$$
\begin{aligned}
\frac{\mathrm{i}}{2}\left(X_{L}^{m}-X_{R}^{m}\right)+\Theta \sigma^{m} \bar{\Theta} & =0 \\
\bar{D} P_{m} & =0 \\
\bar{D}\left(P_{m} \sigma_{\mu \dot{\nu}}^{m} \bar{\Theta}^{\dot{\nu}}\right) & =0
\end{aligned}
$$

where $P_{m}=P_{m}(\tau, \eta, \bar{\eta})$ is a real $N=2$ superfield and $X_{R}^{m}$ is the (antichiral) complex conjugate of $X_{L}^{m}$. The component equation (4.14) then follows directly from (4.15b) and (4.15c). The superfield equations (4.15) may be derived from the $d=3+1$ superparticle action $[6]$

$$
I_{d=3+1}=\int d \tau d \eta d \bar{\eta} P_{m}\left[\frac{i}{2}\left(X_{L}^{m}-X_{R}^{m}\right)+\Theta^{\mu} \sigma_{\mu \dot{\nu}}^{m} \bar{\Theta}^{\dot{\nu}}\right]
$$

This action is invariant both under the local $N=2, d=1$ worldline superconformal transformations (4.14) and also under rigid $N=1, d=3+1$ target spacetime superconformal symmetry.

5. $d=5+1$

In $d=5+1$, the $\operatorname{Spin}(1,5)$ covering group of the Lorentz group is isomorphic to $S L(2, \mathbb{H})$. This group can be defined as the group of complex unimodular $4 \times 4$ matrices $e^{\mu}{ }_{\alpha}$ subject to the pseudoreality condition

$$
\overline{e^{\mu}}=C^{\dot{\mu}}{ }_{\nu} e_{\beta} C^{-1 \beta}{ }_{\dot{\alpha}}
$$

where $C$ is the $d=5+1$ charge conjugation matrix. We work here with the chiral (fundamental) representation. In $d=5+1$, there are no ordinary Majorana spinors, but since complex conjugation does not produce an inequivalent representation in this dimension, linear combinations of spinors and their complex conjugates are covariant. In this way, one may rewrite an ordinary Weyl spinor as an $S U(2)$-Majorana spinor, satisfying

$$
\overline{\psi^{\mu}}{ }_{i}=C^{\dot{\mu}} \epsilon^{i j} \psi_{j}^{\nu}
$$

In this basis, the charge conjugation matrix takes the form

$$
C^{\dot{\mu}}{ }_{\nu}=\left(\begin{array}{cccc}
0 & -1 & 0 & 0 \\
1 & 0 & 0 & 0 \\
0 & 0 & 0 & -1 \\
0 & 0 & 1 & 0
\end{array}\right)
$$

In constructing the coset (1.6) in the $d=5+1$ case using the spinor representation, the maximal subgroup $\mathcal{H}_{B}$ is $[S U(2) \times \widetilde{S U(2)} \times S O(1,1)] \otimes\left\{K_{\tilde{m}}\right\}$. As in the $d=2+1$ and $d=3+1$ 
cases, the $\mathcal{H}_{B}$ subgroup may be represented by block upper triangular matrices, where the blocks are themselves $2 \times 2$ matrices - the quaternions of $S L(2, \mathbb{H})$. When decomposed with respect to the $\mathcal{H}_{B}$ subgroup, the index $\alpha$ on the matrix $e^{\mu}{ }_{\alpha}$ becomes reducible:

$$
e_{\alpha}^{\mu}=\left(\psi_{A}^{\mu}, \phi_{\dot{A}}^{\mu}\right)
$$

where $A$ and $\dot{A}$ correspond respectively to $S U(2)$ and $\widetilde{S U(2)}$. The spinors $\psi^{\mu}{ }_{A}$ and $\phi^{\mu}{ }_{\dot{A}}$ in (5.4) satisfy the $S U(2)$-Majorana condition (5.2) and the whole matrix $e^{\mu}{ }_{\alpha}$ must be unimodular. In terms of $\psi^{\mu}{ }_{A}$ and $\phi^{\mu}{ }_{A}$, the unimodularity condition becomes

$$
\epsilon_{\mu \nu \rho \sigma} \psi^{\mu}{ }_{A} \psi_{B}^{\nu} \phi_{\dot{C}}^{\rho} \phi_{\dot{D}}^{\sigma}=\epsilon_{A B} \epsilon_{\dot{C} \dot{D}} .
$$

It follows from the unimodularity condition (5.5) that neither $\psi^{\mu}{ }_{A}$ nor $\phi^{\mu}{ }_{\dot{A}}$ can vanish.

The $\mathcal{H}_{B}$ gauge transformations of the components of (5.4) are given by

$$
\begin{aligned}
& \delta \psi^{\mu}{ }_{A}=\omega \psi^{\mu}{ }_{A}+\omega_{(A B)} \epsilon^{B C} \psi^{\mu}{ }_{C} \\
& \delta \phi_{\dot{A}}^{\mu}=-\omega \phi_{\dot{A}}^{\mu}+\omega_{\dot{A} \dot{B}} \epsilon^{\dot{B} \dot{C}} \phi_{{ }_{C}}^{\mu}+k_{A \dot{A}} \epsilon^{A B} \psi_{B}^{\mu} .
\end{aligned}
$$

Upon use of the unimodularity condition (5.5) and elimination of gauge components using the $\omega_{\dot{A} \dot{B}}$ and $k_{A \dot{A}}$ transformations in (5.6b), all of the components of $\phi^{\mu}{ }_{\dot{A}}$ may be considered to be dependent. The independent components then live in $\psi^{\mu}{ }_{A}$; but of them half still transform under the $S U(2)$ and $S O(1,1)$ scale transformations. Instead of eliminating three components of $\psi^{\mu}{ }_{A}$ using the $S U(2)$, one may instead make the $S U(2)$ gauge-invariant construction

$$
v^{\mu \nu}=\psi_{A}^{\mu} \psi_{B}^{\nu} \epsilon^{A B} .
$$

This antisymmetric object in two spinorial indices is equivalent to an $S O(1,5)$ vector,

$$
v^{m}=\gamma_{\mu \nu}^{m} v^{\mu \nu}
$$

where $v^{m}$ is a real vector as a consequence of the $S U(2)$-Majorana condition (5.2) on $\psi^{\mu}{ }_{A}$. In terms of $v^{\mu \nu}$, this becomes a pseudoreality condition $\overline{v^{\mu \nu}}=C^{\dot{\mu}}{ }_{\rho} C^{\dot{\nu}} v^{\rho \tau}$. The vector $v^{m}$ is also lightlike, since

$$
v^{m} v_{m}=-2 v^{\mu \nu} v^{\rho \sigma} \epsilon_{\mu \nu \rho \sigma}=0,
$$

as can be seen from the fact that the ${ }_{A}$ index on the commuting variable $\psi^{\mu}{ }_{A}$ runs over two values only. Moreover, since $\psi_{A}^{\mu}$ is nonvanishing, the same holds for $v^{m}$. Finally, since we have not yet fixed the $S O(1,1)$ scale transformations, the vector $v^{m}$ is only defined up to a scale. At this point, we have established in the $d=5+1$ case the conditions for the vector $v^{m}$ that were shown in section 2 to define a sphere, so that in the $d=5+1$ case, $v^{m}$ and hence $\psi^{\mu}{ }_{A}$ parametrise the sphere $S^{4}$. 
The spinorial coset parameter $\psi^{\mu}{ }_{A}$ can be used, following the pattern of our earlier discussions, to project the $d=5+1$ spacetime covariant derivative algebra onto the worldline. The rigid spacetime covariant derivative algebra here is

$$
\left\{D_{\mu}^{i}, D_{\nu}^{j}\right\}=2 \mathrm{i} \epsilon^{i j} \gamma_{\mu \nu}^{m} \partial_{m},
$$

where the indices $i, j$ correspond to the $S U(2)_{\text {aut }}$ outer automorphism of the rigid $d=5+1$ supersymmetry algebra. In analogy with the lower dimensional cases, we can now use linearly transforming $\psi^{\mu}{ }_{A}$ to make the projections

$$
\begin{aligned}
D_{A}^{i} & =\psi_{A}^{\mu} D_{\mu}^{i} \\
\partial_{\tau} & =\epsilon^{A B} \psi_{A}^{\mu} \psi_{B}^{\nu} \gamma_{\mu \nu}^{m} \partial_{m} .
\end{aligned}
$$

The resulting algebra is the $N=4, d=1$ extended worldline covariant derivative algebra

$$
\left\{D_{A}^{i}, D_{B}^{j}\right\}=-\mathrm{i} \epsilon^{i j} \epsilon_{A B} \partial_{\tau} .
$$

This algebra has an automorphism group $S U(2) \times S U(2)_{\text {aut }} \times S O(1,1)$, where the first $S U(2)$ operates on the $A, B$ indices and $S U(2)_{\text {aut }}$ operates on the $i, j$ indices. Note that the former is a subgroup of the Borel subgroup $\mathcal{H}_{B}$ (the $\omega_{(A B)}$ transformations in (5.6)), while the latter is inherited from the rigid $S U(2)$ automorphism of the $d=5+1$ spacetime covariant derivative algebra (5.10).

The rigid $N=4$ worldline supersymmetry corresponding to the algebra (5.12) can then be promoted to a local worldline supersymmetry algebra, the $N=4, d=1$ superconformal algebra, in accordance with our earlier discussions. The new feature of the $d=5+1$ case in distinction to our earlier discussions is that the $S U(2)_{\text {aut }}$ group now plays a significant rôle. The worldline superspace coordinates corresponding to (5.12) are $\left(\tau, \eta_{A}^{i}\right)$. The $N=$ $4, d=1$ superconformal transformations may be defined by the property that $D_{A}^{i}$ transforms homogeneously into a matrix contracted with itself, but does not transform into $\partial_{\tau}$. These transformations are given by

$$
\begin{aligned}
\delta \tau & =\Lambda+\frac{1}{2} \eta_{A}^{i} D_{i}^{A} \Lambda \\
\delta \eta_{A}^{i} & =-\mathrm{i} D_{A}^{i} \Lambda,
\end{aligned}
$$

where $\Lambda(\tau, \eta)$ is an arbitrary real superfield parameter (see appendix).

The $\left(\tau, \eta_{A}^{i}\right)$ superspace $\mathbb{R}^{(1 \mid 4)}$ does not have any invariant subspaces akin to $\mathbb{C}^{(1 \mid 1)}$ in the $d=3+1$ case, where invariant here is understood to mean both under the $N=4, d=$ 1 supersymmetry and also under the $S U(2)_{\text {aut }}$, since the spinor derivative $D_{A}^{i}$ carries an irreducible representation under the product of these groups. 
Nonetheless, there is a way to find an invariant superspace with a smaller fermionic coordinate than the $\left(\tau, \eta_{A}^{i}\right)$ superspace [6]. This involves, however, an initial extension of the superspace. The $\left(\tau, \eta_{A}^{i}\right)$ superspace may be viewed as the group manifold of the $N=4, d=1$ super Poincaré group. Alternatively, since the $N=4, d=1$ super Poincaré group has an $S O(4) \simeq S U(2) \times S U(2)_{\text {aut }}$ automorphism, $\mathbb{R}^{(1 \mid 4)}$ may also be viewed as the coset space $\mathcal{E} / \mathcal{F}$, where $\mathcal{E}$ is the $N=4, d=1$ super Poincaré group times $S U(2) \times S U(2)_{\text {aut }}$ and $\mathcal{F}$ is $S U(2) \times S U(2)_{\text {aut }}$. The virtue of this reformulation is that it suggests another coset space, which one may denote $\mathbb{R}_{H}^{1+2 \mid 4}$, defined as the coset $\mathcal{E} / \mathcal{F}^{\prime}$ where $\mathcal{F}^{\prime}=S U(2) \times U(1)_{\text {aut }}$. The notation $\mathbb{R}_{H}^{1+2 \mid 4}$ means that this space has $1+2$ bosonic coordinates and 4 fermionic coordinates. The two extra bosonic coordinates come from the extra $S^{2}=S U(2)_{\text {aut }} / U(1)_{\text {aut }}$ factor.

Unlike $\mathbb{R}^{(1 \mid 4)}$, the extended superspace $\mathbb{R}_{H}^{1+2 \mid 4}$ does have an invariant subspace [6]. One can see this from the fact that the supersymmetry generator $Q_{A}^{i}$ becomes reducible under the smaller $\mathcal{F}^{\prime}$ stability subgroup. The way to define the invariant subspace is to introduce harmonic coordinates $u_{i}^{ \pm}$for the $S^{2}=S U(2)_{\text {aut }} / U(1)_{\text {aut }}$ factor space and then pass to the "analytic basis" variables

$$
\begin{aligned}
\hat{\tau} & =\tau+\frac{\dot{i}}{2} \eta^{A i} \eta_{A}^{j} u_{i}^{+} u_{j}^{-} \\
\eta^{A \pm} & =\eta^{A i} u_{i}^{ \pm} .
\end{aligned}
$$

One can show that the analytic subspace $\hat{\tau}, \eta^{A+}, u_{i}^{ \pm}$is invariant under the full superconformal group provided the harmonics transform as

$$
\delta u_{i}^{+}=\rho^{++} u_{i}^{+}, \delta u_{i}^{-}=0
$$

where $\rho^{++}=\frac{\mathrm{i}}{2} D^{A+} D_{A}^{+} \Lambda$. In the analytic basis, the projected derivative $u_{i}^{+} D_{A}^{i}$ becomes a partial derivative $-\partial / \partial \eta^{A-}$. On the other hand, the covariant harmonic derivative $D^{++}=$ $u_{i}^{+} \partial / \partial u_{i}^{-}$takes the form

$$
D^{++}=u_{i}^{+} \partial / \partial u_{i}^{-}+\frac{\mathrm{i}}{2} \eta^{A+} \eta_{A}^{+} \partial_{\hat{\tau}}+\eta^{A+} \partial / \partial \eta^{A-} .
$$

In terms of the analytic worldline superfields $P_{m}\left(\hat{\tau}, \eta^{+}, u\right), X^{m}\left(\hat{\tau}, \eta^{+}, u\right), \Theta^{\mu+}\left(\hat{\tau}, \eta^{+}, u\right)$ and $N^{++}\left(\hat{\tau}, \eta^{+}, u\right)$, one can write down the superparticle equation of motion as

$$
D^{++} P_{m}=0, D^{++} X^{m}=i \Theta^{+} \gamma^{m} \Theta^{+}, D^{++} N^{++}+\left(N^{++}\right)^{2}=0 .
$$

These can be derived from an following action formulated in the analytic superspace [6]

$$
\begin{aligned}
S=-i \int d u d \hat{\tau} d \eta^{A+} d \eta_{A}^{+}\left[P_{m}\left(D^{++} X^{m}-i \Theta^{+} \gamma^{m} \Theta^{+}\right)\right. & \\
& \left.+P_{\mu}^{-} \Theta^{\mu+}+P^{--}\left(D^{++} N^{++}+\left(N^{++}\right)^{2}\right)\right],
\end{aligned}
$$


where $P_{\mu}^{-}$and $P^{--}$are analytic Lagrange multipliers. The superfield $N^{++}$plays the rôle of a bridge between the worldline harmonics $u_{i}^{ \pm}$and the target space ones $w_{i}^{ \pm}$:

$$
w_{i}^{+}=u_{i}^{+}+N^{++} u_{i}^{-}, w_{i}^{-}=u_{i}^{-} .
$$

The gauge transformations of $N^{++}$are given by $\delta N^{++}=-\rho^{++}$, so the target space harmonics $w_{i}^{ \pm}$are inert under the worldline superconformal group (5.15). The two sets of harmonics do not coincide off shell. However, on shell the superfield $N^{++}$can be completely gauged away, thus identifying $w_{i}^{ \pm}$with $u_{i}^{ \pm}$. As a result of this gauge fixing, $\rho^{++}=0$ and the superfield parameter $\Lambda$ of the $N=4$ superconformal group becomes constrained

$$
D_{i}^{A} D_{A j} \Lambda=0 .
$$

Note that this restriction is consistent with the group composition law (A.8).

6. $d=9+1$

We turn finally to the most interesting case, $d=10$. For $N=1$ spacetime supersymmetry the spinors are Majorana-Weyl, i.e. sixteen-component real Weyl spinors. The spin group, $\operatorname{Spin}(1,9)$, is related to the Lorentz group, $S O^{\uparrow}(1,9)$, via the invariance of the gammamatrices; if $e_{\alpha}^{\mu} \in \operatorname{Spin}(1,9)$ (so that it is a real $16 \times 16$ matrix), one has the constraint

$$
e_{\alpha}^{\mu} e_{\beta}^{\nu}\left(\gamma^{m}\right)_{\mu \nu}=v^{m}{ }_{a}\left(\gamma^{a}\right)_{\alpha \beta}
$$

where $v$ is the corresponding element of $S O^{\uparrow}(1,9)$. The $\gamma$-matrices in (6.1) are symmetric on their spinor indices; they are not Dirac matrices, but are analogs of the $\sigma$-matrices in four dimensions. Spinorial indices cannot be raised or lowered in ten dimensions, but there is another set of matrices $\gamma_{a}^{\alpha \beta}$ with upper indices. ${ }^{*}$ Equation (6.1) can be expanded in a complete set of symmetric $16 \times 16$ matrices consisting of $\left(\gamma^{a}\right)_{\alpha \beta}$ and $\left(\gamma^{a b c d e}\right)_{\alpha \beta}$, the latter being self-dual on its vectorial indices. We get

$$
e^{\mu}{ }_{\alpha} e^{\nu}{ }_{\beta}\left(\gamma^{m}\right)_{\mu \nu}\left(\gamma_{a}\right)^{\alpha \beta}=16 v^{m}{ }_{a}
$$

and

$$
e^{\mu}{ }_{\alpha} e_{\beta}^{\nu}\left(\gamma^{m}\right)_{\mu \nu}\left(\gamma^{a b c d e}\right)^{\alpha \beta}=0 .
$$

* These obey the anticommutation relation $\gamma_{\alpha \beta}^{a} \gamma^{b \beta \gamma}+\gamma_{\alpha \beta}^{b} \gamma^{a \beta \gamma}=2 \eta^{a b} \delta_{\alpha}^{\gamma}$. In a specific choice of spinor basis one may have $\gamma_{\alpha \beta}^{a}=\gamma_{a}^{\alpha \beta}$ numerically and also the values

$$
\gamma_{\alpha \beta}^{0}=\left(\begin{array}{ll}
\mathbb{1} & 0 \\
0 & \mathbb{1}
\end{array}\right), \gamma_{\alpha \beta}^{\tilde{a}}=\left(\begin{array}{cc}
0 & \sigma^{\tilde{a}} \\
\left(\sigma^{\tilde{a}}\right)^{T} & 0
\end{array}\right), \gamma_{\alpha \beta}^{9}=\left(\begin{array}{cc}
-\mathbb{1} & 0 \\
0 & \mathbb{1}
\end{array}\right) .
$$

An important identity is $\gamma_{a \alpha(\beta} \gamma_{\gamma \delta)}^{a}=0$. 
The matrix $e^{\mu}{ }_{\alpha}$, considered as the product of $\overline{\mathbf{1 6}}$ and $\mathbf{1 6}$ representations of $S O(1,9)$, is decomposable into $\mathbf{1}+\mathbf{4 5}+\mathbf{2 1 0}$. Now, eq. (6.3) imposes precisely 210 conditions, while eq. (6.2) is equivalent to the unimodularity condition $(\operatorname{det} e)=1$. The remaining 45 degrees of freedom correspond to parameters of $S O(1,9)$.

The Borel subgroup $H_{B}=\left[S O(8) \times S O^{\uparrow}(1,1)\right] \otimes\left\{K_{\tilde{m}}\right\}$ acts on the $\alpha, \beta$ indices by

$$
e^{\prime \mu}{ }_{\alpha}=e^{\mu}{ }_{\beta} \Omega_{\alpha}^{\beta} .
$$

To describe this action explicitly, it is convenient to split the $\alpha$ index up into its $S O(8)$ irreducible parts, $e_{\alpha}^{\mu}=\left(e_{A}^{\mu}, e^{\mu}{ }_{\dot{A}}\right)$, where $A, \dot{A}=1 \ldots 8$ are correspondingly indices of the $\mathbf{8}_{\mathbf{s}}$ and $\mathbf{8}_{\mathbf{c}}$ fundamental spinor representations of $S O(8)$. The matrix $\Omega$ is block triangular,

$$
\Omega^{\alpha}{ }_{\beta}=\left(\begin{array}{cc}
\Omega^{A}{ }_{B} & \Omega^{A} \dot{B} \\
0 & \Omega^{\dot{A}} \dot{B}
\end{array}\right) .
$$

Here $\Omega^{A}{ }_{B}$ is an $S O(8)$ matrix in the $\mathbf{8}_{\mathbf{s}}$ representation times an $S O(1,1)$ scale factor, $\Omega^{\dot{A}}{ }_{\dot{B}}$ is the same $S O(8)$ matrix in the $\mathbf{8}_{\mathbf{c}}$ representation times the opposite $S O(1,1)$ factor, i.e. infinitesimally we have

$$
\Omega_{B}^{A} \simeq \delta^{A}{ }_{B}(1+\omega)+\omega_{B}^{A}, \quad \Omega_{\dot{B}}^{\dot{A}} \simeq \delta_{\dot{B}}^{\dot{A}}(1-\omega)+\omega^{\dot{A}}{ }_{\dot{B}},
$$

and the $K_{\tilde{m}}$ parameter $\Omega_{\dot{B}}^{A}$ corresponds to the eight-dimensional vector $\mathbf{8}_{\mathbf{v}}$ representation of $S O(8)$.

Thus, under an $H_{B}$-transformation $e^{\mu}{ }_{A}$ transforms homogeneously while $e^{\mu}{ }_{A}$ transforms into $e_{A}^{\mu}$ as well as into itself. The constraints (6.2), (6.3) together with the gauge transformations (6.4) imply that the components of $e^{\mu}{ }_{\dot{A}}$ are either gauge degrees of freedom or are functions of the components of $e^{\mu}{ }_{A}$. We can thus focus on $e^{\mu}{ }_{A}$. From (6.1) and the values given for $\left(\gamma^{a}\right)_{A B}$ we have

$$
\begin{aligned}
e^{\mu}{ }_{A} e_{B}^{\nu}\left(\gamma^{m}\right)_{\mu \nu} & =v^{m}{ }_{a}\left(\gamma^{a}\right)_{A B}=v^{m}-\left(\gamma^{-}\right)_{A B} \\
& =\sqrt{2} v^{m}{ }_{-} \delta_{A B}
\end{aligned}
$$

where we have introduced a light-cone decomposition of the vector index, $\gamma^{a}=\left(\gamma^{-}, \gamma^{\tilde{a}}, \gamma^{+}\right)$. The vector $v^{m}{ }_{-}=\frac{1}{8 \sqrt{2}} e^{\mu}{ }_{A} e^{\nu}{ }_{B}\left(\gamma^{m}\right)_{\mu \nu} \delta^{A B}$ is lightlike and invariant under the $S O(8) \otimes\left\{K_{\tilde{m}}\right\}$ subgroup of $H_{B}$. The independent coset space parameters are contained in the vector $v^{m}$ which is defined up to a positive scale transformation. Hence the coset is the eight-sphere $S^{8}$.

The matrix $e^{\mu}{ }_{A}$ can be used to project from $d=10$ superspace onto the worldline. The flat $d=10$ covariant derivatives $D_{\mu}, \partial_{m}$ obey

$$
\left\{D_{\mu}, D_{\nu}\right\}=2 i\left(\gamma^{m}\right)_{\mu \nu} \partial_{m}
$$


and projected covariant derivatives on the worldline can be defined by

$$
\begin{aligned}
D_{A} & =e^{\mu}{ }_{A} D_{\mu} \\
\partial_{\tau} & =\frac{1}{8} e^{\mu}{ }_{A} e^{\nu}{ }_{A}\left(\gamma^{m}\right)_{\mu \nu} \partial_{m} .
\end{aligned}
$$

The projected derivatives satisfy the $N=8, d=1$ extended worldline covariant derivative algebra

$$
\left\{D_{A}, D_{B}\right\}=2 i \delta_{A B} \partial_{\tau}
$$

It is not known what the $N=8$ worldline supersymmetric action is for the $d=10$ superparticle, but solutions to the equations of motion can be written down in a way that is manifestly $N=8$ worldline supersymmetric. On the space $\mathcal{M} \times \operatorname{Spin}(1,9)(\mathcal{M}=\operatorname{superspace})$, introduce new coordinates $x^{a}=x^{m} v^{a}{ }_{m}, \theta^{\alpha}=\theta^{\mu} e^{\alpha}{ }_{\mu}$ together with the coset coordinates $e^{\mu}{ }_{\alpha}$, where $v^{a}{ }_{m}$ and $e^{\alpha}{ }_{\mu}$ are the inverses of $v^{m}{ }_{a}$ and $e^{\mu}{ }_{\alpha}$. Then a super-lightlike line in $\mathcal{M}$ passing through the origin is described by the $H_{B}$-covariant equations

$$
x^{+}=x^{\tilde{a}}=\theta^{\dot{A}}=0 .
$$

Equivalently, we can write

$$
\begin{aligned}
x^{m} & =x^{-} v^{m}- \\
\theta^{\mu} & =\theta^{A} e^{\mu}{ }_{A}
\end{aligned}
$$

with

$$
v_{-}^{m}=\frac{1}{8 \sqrt{2}} e_{A}^{\mu} e_{A}^{\nu}\left(\gamma^{m}\right)_{\mu \nu} .
$$

Identifying the worldline parameters $\left(\tau, \eta^{A}\right)$ with $\left(x^{+}, \theta^{A}\right)$ we can see that $(6.12)$ is a solution of the differential equations

$$
\begin{aligned}
D_{A} X^{m}-i D_{A} \Theta^{\mu}\left(\gamma^{m}\right)_{\mu \nu} \Theta^{\nu} & =0 \\
D_{A} \Theta^{\mu} & =e^{\mu}{ }_{A},
\end{aligned}
$$

where $X^{m}\left(\tau, \eta^{A}\right)$ and $\Theta^{\mu}\left(\tau, \eta^{A}\right)$ are now $N=8$ worldline superfields and $e^{\mu}{ }_{A}$ is constant. Eq. (6.12) gives the solution of (6.14) up to a (supersymmetric) shift of origin. Note that these $d=10$ equations are not target space superconformally invariant, although the corresponding equations of motion (and actions) are for $d=3,4$ and 6 . If one defines the superconformal group of the superspace as the group of transformations which scale the line element, then in $d=10$ this group consists only of super-Poincaré and scale transformations. In order to include conformal boosts it would be necessary to extend the bosonic part of the algebra; such extensions exist abstractly but cannot be realised on ordinary superspace.

It is interesting to relate our new formalism to other approaches in the literature. From (6.7) we can see that any of the $e_{A}^{\mu}, A=1, \ldots 8$ determines the same lightlike vector. 
Indeed, we can take the independent parameters of the coset $S^{8}$ to be contained in the sixteen-component spinor $e^{\mu}{ }_{1} \equiv \psi^{\mu}$. The space of $\psi^{\mu}$ modulo scale transformations is $S^{15}$ and is related to $S^{8}$ by the Hopf fibration, which has fibre $S^{7}$. From the transformation (see $(6.4)-(6.6))$

$$
\delta e^{\mu}{ }_{A}=e^{\mu}{ }_{A} \omega+e^{\mu}{ }_{B} \omega^{B}{ }_{A}
$$

one finds

$$
\delta \psi^{\mu}=\psi^{\mu} \omega+e^{\mu}{ }_{A} \omega^{A}{ }_{1} .
$$

The $S O(8)$ algebra matrix $\omega_{B}^{A}=\omega_{A B}$ is antisymmetric, $\omega_{A B}=-\omega_{B A}$. Hence $\omega^{A}{ }_{1}$ corresponds, in fact, to seven parameters $\omega^{\hat{A}} 1, \hat{A}=2, \ldots 8$. Without losing generality one can make a field-dependent change of transformation parameter, analogously to eq. (3.19),

$$
\omega_{\hat{A} 1}=\xi_{\nu} e_{\hat{A}}^{\nu}
$$

where $\xi_{\nu}$ is an arbitrary commuting $S O(1,9)$ spinor parameter. Substituting this back into (6.15) and using the identities $e^{\nu}{ }_{\hat{A}} e^{\mu}{ }_{\hat{A}}=e^{\nu}{ }_{A} e^{\mu}{ }_{A}-e^{\nu}{ }_{1} e^{\mu}{ }_{1}$ and

$$
e^{\nu}{ }_{A} e^{\mu}{ }_{A}=\frac{1}{16}\left(\gamma_{m}\right)^{\mu \nu} e^{\rho}{ }_{A}\left(\gamma^{m}\right)_{\rho \sigma} e^{\sigma}{ }_{A}
$$

(which is just equivalent to the condition $e^{\mu}{ }_{\alpha} e^{\nu}{ }_{\beta}\left(\gamma_{a}\right)^{\alpha \beta}=v^{m}{ }_{a}\left(\gamma_{m}\right)^{\mu \nu}$ conjugate to (6.1), taken in the - projection of $a$ ) and also

$$
e^{\rho}{ }_{A}\left(\gamma^{m}\right)_{\rho \sigma} e^{\sigma}{ }_{A}=8 e^{\rho}{ }_{1}\left(\gamma^{m}\right)_{\rho \sigma} e^{\sigma}{ }_{1}=8 \psi^{\rho}\left(\gamma^{m}\right)_{\rho \sigma} \psi^{\sigma},
$$

we finally get

$$
\delta \psi^{\mu}=\omega^{\prime} \psi^{\mu}+\frac{1}{2}\left(\gamma_{m}\right)^{\mu \nu} \xi_{\nu} \psi \gamma^{m} \psi .
$$

with the scale factor $\omega^{\prime}=\omega-\xi_{\nu} \psi^{\nu}$.

The transformation (6.20) is precisely that used by Berkovits in his approach to the superparticle [7]. Note that as a result of the field-dependent change (6.17), the gauge transformations (6.4) are realised in (6.20) nonlinearly. Moreover, the 16-dimensional parameter $\xi_{\nu}$ introduced in (6.17) instead of the 7-dimensional one $\omega_{\hat{A} 1}$ is defined also up to a pregauge freedom $[7,5]$

$$
\delta \xi_{\mu}=\left(\gamma^{m}\right)_{\mu \nu} \psi^{\nu} r^{m}
$$

with a 10-dimensional parameter $r^{m}$ which is defined up to the transformation

$$
\delta r^{m}=\left(\gamma^{m}\right)_{\mu \nu} \psi^{\mu} \psi^{\nu} q
$$

where $q$ is a scalar parameter. 
In fact, it is possible to generalise Berkovits' formalism in an $S O(8)$ covariant fashion by using $e_{A}^{\mu}$ instead of $\psi^{\mu}=e^{\mu}{ }_{1}$. One could then solve for $p^{m}$ as $p^{m}=e^{\mu}{ }_{A}\left(\gamma^{m}\right)_{\mu \nu} e^{\nu}{ }_{A}$ and introduce the additional variables

$$
\begin{aligned}
f_{\mu A} & =x^{m}\left(\gamma_{m}\right)_{\mu \nu} e_{A}^{\nu}+\mathrm{i}\left(\gamma^{m}\right)_{\mu \nu} \theta^{\nu} e_{A}^{\rho}\left(\gamma_{m}\right)_{\rho \sigma} \theta^{\sigma}, \\
\lambda_{A}^{m} & =\theta^{\mu}\left(\gamma^{m}\right)_{\mu \nu} e_{A}^{\nu} .
\end{aligned}
$$

The Brink-Schwarz Lagrangian can then be rewritten in terms of the constrained variables $\left(e^{\mu}{ }_{A}, f_{\mu A}, \lambda_{A}^{m}\right)$. Alternatively, one can use the inverse group element $e_{\mu}^{\alpha}=\left(e_{\mu}^{A}, e_{\mu}^{\dot{A}_{\mu}}\right)$ and write $p^{m}=e_{\mu}^{\dot{A}}\left(\gamma^{m}\right)^{\mu \nu} e_{\nu}^{\dot{A}}$. The additional variables are then

$$
\begin{aligned}
f^{\mu \dot{A}} & =x^{m}\left(\gamma_{m}\right)^{\mu \nu} e_{\nu}^{\dot{A}}+\mathrm{i} \theta^{\mu} \theta^{\nu} e_{\nu}^{\dot{A}}, \\
\lambda^{\dot{A}} & =\theta^{\mu} e_{\mu}^{\dot{A}},
\end{aligned}
$$

the complete final set of constrained variables being $\left(e_{\mu}^{\dot{A}}, f^{\mu \dot{A}}, \lambda^{\dot{A}}\right)$. This option does not exist in $d=3,4$ where a Berkovits-type formalism would differ from the supertwistor one solely in its choice of a Grassmann-odd variable instead of a Grassmann-even one. In $d=6$, one choice of chirality in the solution for $p^{m}$ leads to a Berkovits-type formalism, whereas the other choice gives rise to a supertwistor formalism [17].

Finally, we note that our Lorentz-coset formalism also allows for pure spinors. If we set $\psi^{\mu}=e^{\mu}{ }_{1}$ and $\phi^{\mu}=e^{\mu}{ }_{2}$ it is easy to show that $\chi^{\mu}=\psi^{\mu}+i \phi^{\mu}$ is pure, i.e. it obeys the constraint

$$
\chi^{\mu}\left(\gamma^{m}\right)_{\mu \nu} \chi^{\nu}=0
$$

It has been suggested that pure spinors might play a rôle in ten-dimensional supergeometry as it is possible to understand the constraints of supergravity and super Yang-Mills theories as arising from integrability along "pure spinor lines" [12]. The space of pure spinors is the twenty-two dimensional coset $S O^{\uparrow}(1,9) / S U(4) \otimes\left\{K_{\tilde{m}}\right\}$ [18]. This space is non-compact, but the space of projective pure spinors, $S O^{\uparrow}(1,9) /\left[U(4) \times S O^{\uparrow}(1,1)\right] \otimes\left\{K_{\tilde{m}}\right\}$, is a compact space with twenty real dimensions. Locally this space can be viewed as $S^{8} \times S O(8) / U(4)$; globally it is the bundle of complex structures preserving the metric on $S^{8}$ for which $S O(1,9)$ acts as the conformal group. (These complex structures are only locally defined since $S^{8}$ is not a complex manifold.) This picture leads to the possibility that the worldline coset for $N=8$ might be $S O(8) / U(4)$, and that the target space coset might be extended to the space of projective pure spinors. This would be a natural extension of the $N=4$ case, since there $S U(2) / U(1)=S O(4) / U(2)$. 


\section{Conclusions}

In this paper we have interpreted the additional "twistor-like" variables occurring in the STV version of the superparticle as parameters of the coset spaces $S O^{\uparrow}(1, d-1) / \mathcal{H}_{B}$. We have seen that this description is valid for $d=3,4,6$ at the level of the action and also for $d=10$ on-shell. Although we have focused on flat target spaces, it is straightforward to extend this formalism to include non-trivial supergravity and super-Maxwell background fields [6]. In that case, demanding that the projected derivatives satisfy a flat superalgebra on the worldline puts constraints on the backgrounds, and these constraints correspond to those derivable from lightlike integrability $[10,11]$. For example, in the $d=10$ Maxwell case, lightlike integrability implies the equations of motion for the Maxwell multiplet.

The STV variables appear in the $\eta$-expansion of the worldline spinorial superfields $\Theta$, and have not been introduced into the target space as independent variables. Although it should be possible to extend the target space to incorporate them independently, it has not been necessary to do so in the $d=3,4 \& 6$ cases in order to have a satisfactory off-shell formulation, as we have seen. Indeed, although we introduced both worldline and target space harmonic variables in the $d=6$ case (following [6]), it is not obligatory to do so since the model can be formulated off-shell in terms of constrained $4+4$ component worldline $N=4$ superfields. On the other hand, it is likely that additional variables will be necessary for the $d=10$ case. Since there is no internal symmetry group in $d=10$, these new variables should parametrise some coset of the Lorentz group. In this paper we have seen that a candidate coset is the eight-sphere, which has the advantage of being compact, in contradistinction to the previously proposed light-cone harmonic superspace $[14,15]$. However, as we have remarked, there are also other compact coset spaces such as the space of projective pure spinors that could play a rôle in the off-shell formulation of the $d=10$ superparticle.

Although we have not succeeded in giving a fully satisfactory off-shell account of the $d=10$ superparticle, we believe that the compact coset space approach will be of central importance to this problem. The basic idea behind the STV and supertwistor approaches to the superparticle is to solve the constraint $p^{2}=0$ by using spinor variables. If this is done

with a single minimal spinor $\psi,\left(p \sim \psi^{2}\right)$, then the space of lightlike vectors up to a scale can be interpreted in terms of the Hopf fibrations, i.e. the spheres of real spinors up to a positive scale $S^{1}, S^{3}, S^{7}, S^{15}$ are fibre bundles over the celestial spheres $S^{1}, S^{2}, S^{4}, S^{8}$ with fibres $1, S^{1}, S^{3}, S^{7}$. The main difference in the $d=10$ case is that $S^{7}$ is not a group. This is an advantage of the coset approach, which guarantees that the divisor gauge transformations form a group. Actually, as we have seen, the $\operatorname{Spin}(1, d-1) / \mathcal{H}_{B}$ coset can be parametrised by the first column of a group element in $d=3,4$ and by a pseudo-real spinor for $d=6$, so that in these three cases we get back directly to the Hopf fibration picture. However, in $d=10$, we need the $16 \times 8$ matrix $e^{\mu}{ }_{A}$ which is constrained by $(6.7),(6.18)$ so that the situation is somewhat different. 
Another way of looking at the Hopf fibrations is via the division algebras. The celestial spheres can be presented as $K P^{1}$ with $K=\mathbb{R}, \mathbb{C}, \mathbb{H}, \mathbb{O}$ for $d=3,4,6,10$ respectively. The division algebra approach to supersymmetry in these dimensions has been studied by many authors (see for instance $[19,17]$ ) and in some sense the supergeometries for both the worldline and the target superspaces can be considered as real, complex and quaternionic for $d=3,4,6$ respectively. The open problem of $d=10$ can therefore be thought of as the problem of finding out how octonionic supergeometries should be defined. This problem is certainly relevant to finding the $d=10$ action, for in $d=4$ and $d=6$ it is necessary to compexify and "quaternionify" (or rather "harmonise") the even part of the target superspaces in order to go off shell.

Finally, we note that although the superparticle has proved to be a surprisingly difficult and interesting problem, its solution should be seen as only a step on the way to understanding the supergeometry of ten-dimensional superspace and of the Green-Schwarz superstring*. We believe that the coset method presented here may be useful tool for tackling these questions.

\section{Acknowledgements}

A.G. would like to thank sincerely F. Delduc, B. de Wit, O.V. Ogievetsky, V.I. Ogievetsky and E. Sokatchev for valuable discussions and the Theoretical Physics group of Imperial College for its warm hospitality.

\section{Appendix. Superconformal groups in one dimension}

Here we give some details of the worldline superconformal groups. We start with the $N$-extended Poincaré supersymmetry algebra in one dimension

$$
\left\{Q_{a}, Q_{b}\right\}=2 \delta_{a b} P, \quad(a, b=1,2, \ldots, N)
$$

It possesses an $O(N)$ automorphism group with $Q_{a}$ being a vector, and $P$ a singlet. It can be realised in a real superspace $R^{(1 \mid N)}:\left(\tau, \eta^{a}\right)$ with a single bosonic coordinate $\tau$ and $N$ Grassmann coordinates $\eta^{a}$. The covariant Grassmann derivatives

$$
D_{a}=\frac{\partial}{\partial \eta^{a}}+\mathrm{i} \eta_{a} \partial_{\tau}
$$

satisfy the algebra

$$
\left\{D_{a}, D_{b}\right\}=2 \mathrm{i} \partial_{\tau}
$$

\footnotetext{
* In a recent interesting paper [20], the $\kappa$-symmetry of the $d=4$ Green-Schwarz superstring is explained as a world-sheet supersymmetry.
} 
Under the super worldline diffeomorphisms $\tau^{\prime}=\tau+\delta \tau(\tau, \eta), \eta^{\prime}=\eta+\delta \eta(\tau \eta)$, the Grassmann derivatives $D_{a}$ will in general transform into themselves and $\partial_{\tau}$ :

$$
\delta D_{a} \equiv D_{a}^{\prime}-D_{a}=-\left(D_{a} \delta \eta_{b}\right) D_{b}+\left[\mathrm{i}\left(D_{a} \delta \eta_{b}\right) \eta_{b}+\mathrm{i} \delta \eta_{a}-D_{a} \delta \tau\right] \partial_{\tau}
$$

Hence $D_{a}$ will transform homogeneously provided the following constraint is imposed on the parameters $\delta \tau$ and $\delta \eta$

$$
\mathrm{i}\left(D_{a} \delta \eta_{b}\right) \eta_{b}+\mathrm{i} \delta \eta_{a}-D_{a} \delta \tau=0
$$

The corresponding constrained transformations are the superconformal ones. The constraint (A.5) can be equivalently derived from the requirement that the line element $d s=d \tau+\mathrm{i} \eta_{a} d \eta_{a}$ transform into itself, i.e. $\delta d s \sim d s$.

The general solution to the constraint $(A .5)$ is expressed in terms of a real scalar superfield $\Lambda(\tau, \eta)$

$$
\delta \eta_{a}=-\frac{\mathrm{i}}{2} D_{a} \Lambda, \quad \delta \tau=\Lambda-\frac{1}{2} \eta_{a} D_{a} \Lambda
$$

Then the transformation laws of the line element and covariant derivatives are given by

$$
\begin{aligned}
\delta d s & =\left(\partial_{\tau} \Lambda\right) d s \\
\delta D_{a} & =-\frac{1}{2}\left(\partial_{\tau} \Lambda\right) D_{a}+\frac{\mathrm{i}}{4}\left[D_{a}, D_{b}\right] \Lambda D_{b}
\end{aligned} .
$$

Furthermore, the commutator of two transformations (A.6) yields a transformation withe the bracket parameter

$$
\Lambda_{\mathrm{br}}=\Lambda_{2} \partial_{\tau} \Lambda_{1}-\Lambda_{1} \partial_{\tau} \Lambda_{2}+\frac{\mathrm{i}}{2} D_{a} \Lambda_{1} D_{a} \Lambda_{2}
$$

Now, let us characterise some specific features of the cases $N=1,2,4,8$.

For $\mathbf{N}=\mathbf{1}$ the superfield $\Lambda(\tau, \eta)=\lambda(\tau)+2 \mathrm{i} \eta \epsilon(\tau)$ contains the irreducible $(1+1)$ representation of $\mathrm{N}=1$ supersymmetry, with $\lambda$ and $\epsilon$ being translation and supertranslation parameters.

For $\mathbf{N}=\mathbf{2} \Lambda(\tau, \eta)$ is also irreducible. It describes the $(2+2)$ multiplet

$$
\Lambda=\lambda+2 \mathrm{i} \eta_{a} \epsilon_{a}+\mathrm{i} \eta_{1} \eta_{2} \rho
$$

where $\rho(\tau)$ corresponds to local $S O(2)$ rotations. This group possesses a natural holomorphic structure. To see this one should pass to the "eigenvectors" of $S O(2)=U(1)$,

$$
\begin{gathered}
\eta=\frac{1}{\sqrt{2}}\left(\eta_{1}+\mathrm{i} \eta_{2}\right), \bar{\eta}=\frac{1}{\sqrt{2}}\left(\eta_{1}-\mathrm{i} \eta_{2}\right) \\
D=\frac{1}{\sqrt{2}}\left(D_{1}-\mathrm{i} D_{2}\right)=\frac{\partial}{\partial \eta}+\mathrm{i} \bar{\eta} \partial_{\tau}, \quad \bar{D}=\frac{1}{\sqrt{2}}\left(D_{1}+\mathrm{i} D_{2}\right)=\frac{\partial}{\partial \bar{\eta}}+\mathrm{i} \eta \partial_{\tau} .
\end{gathered}
$$


The derivative $\bar{D}$ is nilpotent, $\bar{D}^{2}=0$, so there exists a basis in $R^{(1 \mid 2)}$ where it is simply a partial derivative. This is the chiral basis

$$
\left\{\tau_{L}=\tau+\mathrm{i} \eta \bar{\eta}, \eta, \bar{\eta}\right\} ; \quad(\bar{D})_{L}=\frac{\partial}{\partial \eta}
$$

One can check that the chiral subspace $\left(\tau_{L}, \eta\right)$ is closed under the superconformal transformations (A.6); for instance, $\delta \eta=-\frac{\mathrm{i}}{2} \bar{D} \Lambda, \quad \bar{D} \delta \eta=0$.

In the $\mathbf{N}=\mathbf{4}$ case the scalar superfield

$$
\begin{gathered}
\Lambda(\tau, \eta)=\lambda+2 \mathrm{i} \eta_{a} \epsilon_{a}+\frac{\mathrm{i}}{2} \eta_{a} \eta_{b} \rho_{a b}+\eta_{a}^{3} \xi_{a}+\eta^{4} k \\
\eta_{a}^{3}=\frac{1}{3 !} \epsilon_{a b c d} \eta_{b} \eta_{c} \eta_{d}, \quad \eta^{4}=\frac{1}{4 !} \epsilon_{a b c d} \eta_{a} \eta_{b} \eta_{c} \eta_{d},
\end{gathered}
$$

describes the reducible $(8+8)$ representation of $N=4$ supersymmetry. Its irreducible part can be obtained by imposing a self-duality constraint

$$
\left[D_{a}, D_{b}\right] \Lambda=\frac{1}{2} \epsilon_{a b c d}\left[D_{c}, D_{d}\right] \Lambda
$$

A nontrivial property of this constraint is its consistency with the group composition law (A.8), i.e., the bracket parameter $(A .10) \Lambda_{\mathrm{br}}$ satisfies the same constraint. The constraint (A.13) has a transparent meaning: in the $N=4$ case the automorphism group $S O(4)$ is (locally) equivalent to the product of two $S U(2)$ groups, $S O(4) \sim S U(2) \times S U(2)_{\mathcal{A}}$, and the transformations of one of them, say $S U(2)_{\mathcal{A}}$, are fixed by $(A .13)$.

Passing to the $S U(2) \times S U(2)_{\mathcal{A}}$ spinor notation ${ }^{\dagger}, \quad \eta_{a} \rightarrow \eta_{A i}, D_{a} \rightarrow D_{A i}=\partial / \partial \eta^{A i}+$ $\frac{1}{2} \mathrm{i} \eta_{A i} \partial_{\tau}$, one can rewrite the commutation relations $(A .3)$ as

$$
\left[D_{A i}, D_{B j}\right]=2 \mathrm{i} \epsilon_{A B} \epsilon_{i j} \partial_{\tau}
$$

and the constraint $(A .13)$ as

$$
D_{i}^{A} D_{A j} \Lambda=0
$$

Owing to the presence of the two $S U(2)$ automorphism groups, the algebra $(A .14)$ is very similar to the algebra of $N=2, d=6$ supersymmetry. As in the latter case, it is useful to introduce $S U(2)_{\mathcal{A}} / U(1)$ harmonics $u_{i}^{ \pm}, u^{+i} u_{i}^{-}=1$ in order to convert all explicit $S U(2)_{\mathcal{A}}$ indices into $U(1)$ charges: $D_{A i} \rightarrow D_{A}^{ \pm}=u_{i}^{+} D_{A}^{i}, \eta^{A i} \rightarrow \eta^{A \pm}=u_{i}^{+} \eta^{A i}$. In the extended superspace $\tau, \eta, u$ one can then pass to an analytic basis

$$
\hat{\tau}=\tau+\frac{\mathrm{i}}{2} \eta^{A i} \eta_{A}^{j} u_{i}^{+} u_{j}^{-}, \eta^{A \pm}=u_{i}^{+} \eta^{A i}, u_{i}^{ \pm}
$$

$\dagger \quad$ Here $A=1,2$ and $i=1,2$ are indices of $S U(2)$ and $S U(2)_{\mathcal{A}}$ respectively. The spinor indices are raised and lowered as usual with the help of the $\epsilon_{A B}$ and $\epsilon_{i j}$ symbols, e.g. $\eta_{A i}=\epsilon_{A B} \epsilon_{i j} \eta^{B j}$. 
and find that the extended superspace contains an analytic subspace of smaller Grassmann dimension with coordinates

$$
z_{A}=\left(\hat{\tau}, \eta^{A+}, u\right)
$$

The analytic superfields $\Phi\left(\hat{\tau}, \eta^{A+}, u\right)$ automatically satisfy the constraint $D_{A}^{+} \Phi=0$, because in the analytic basis $(A .16) D_{A}^{+}$becomes just $-\partial / \partial \eta^{A-}$.

An important property of the analytic subspace $(A .17)$ is its closure under the full unconstrained $N=4$ superconformal group (A.6), provided that the harmonics transform as follows

$$
\delta u_{i}^{+}=\rho^{++} u_{i}^{-}, \delta u_{i}^{-}=0, \rho^{++}=\frac{\mathrm{i}}{2} D^{A+} D_{A}^{+} \Lambda .
$$

Then the other variations are analytic as well,

$$
\delta \hat{\tau}=-\frac{1}{4} D^{A+} D_{A}^{+}\left(\eta^{B-} \eta_{B}^{-} \Lambda\right), \delta \eta_{A}^{+}=\frac{\mathrm{i}}{2} D^{B+} D_{B}^{+}\left(\eta_{A}^{-} \Lambda\right)
$$

because the third power of $D^{+}$vanishes automatically.

The analytic parameters are not arbitrary, but are constrained to satisfy

$$
D^{++} \rho^{++}=0, D^{++} \delta \hat{\tau}=\mathrm{i} \eta^{A+} \delta \eta_{A}^{+}, D^{++} \delta \eta_{A}^{+}=\rho^{++} \eta_{A}^{+}
$$

Here $D^{++}=u_{i}^{+} \partial / \partial u_{i}^{-}+\frac{\mathrm{i}}{2} \eta^{A+} \eta_{A}^{+} \partial_{\hat{\tau}}$ is one of the covariant derivatives on the sphere $S^{2}=$ $S U(2)_{\mathcal{A}} / U(1)$. Note, that the subgroup $(A .13)$ is obtained simply by putting $\rho^{++}=0$.

An interesting feature of the transformations $(A .18),(A .19)$ is that they preserve the supervolume of the analytic subspace $(A .17)$ :

$$
\frac{\partial}{\partial \hat{\tau}} \delta \hat{\tau}+u_{i}^{-} \frac{\partial}{\partial u_{i}^{+}}-\frac{\partial}{\partial \eta^{A+}} \delta \eta^{A+}=0
$$

Finally we turn to the $N=8$ case. Here, one encounters difficulty even defining an appropriate worldline superconformal algebra. In this case, the general unconstrained superfield $\Lambda(\tau, \eta)$ describing $(128+128)$ degrees of freedom is a highly reducible representation of $N=8$ supersymmetry. Following the $N=4$ case, it is natural to ask whether there are any representations aside from the full $\Lambda$ itself, that are consistent with the group composition law (A.8).

The only $S O(8)$ covariant constraint one can impose on $\Lambda$ without putting it on shell is the fourth-order self-duality constraint

$$
D_{a} D_{b} D_{b} D_{b} \Lambda=\frac{1}{4 !} \epsilon_{a b c d e f g h} D_{e} D_{f} D_{g} D_{h} \Lambda
$$

However, this constraint is not compatible with $(A .8)$. Therefore in order to constrain $\Lambda$ one would have to explicitly break the tangent group $S O(8)$ symmetry by restricting the $S O(8)$ 
parameter $\left[D_{a}, D_{b}\right] \Lambda$. Now one can easily prove a lemma: if the surviving tangent group is a subgroup of $S O(7)$, then this would put $\Lambda$ on shell, i.e., such a restriction would be too strong. Indeed, the constraint corresponding to a surviving $S O(7)$ tangent group reads $D_{1} D_{r} \Lambda=0$, where $r=2, \ldots, 8$ (the 28 parameters of $S O(8)$ are decomposed with respect to $S O(7)$ as $\mathbf{2 1 + 7}$ and the $\mathbf{7}$ is set to zero). However, acting on this constraint with $D_{1}$ one would get the on-shell condition $\partial_{\tau} D_{r} \Lambda=0$ and hence an unwanted restriction on the ordinary worldline supersymmetry. Therefore the sought-for tangent group should not be a subgroup of $S O(7)$. This, in particular, excludes all of the simple proper subgroups of $S O(8)$. We will not go into further detail here, but mention only that the breaking of $S O(8)$ down to a nonsimple subgroup $S O(6) \times S O(2)$ that has been suggested in the literature also puts $\Lambda$ on shell. Thus, whether it is possible to formulate an appropriate $N=8$ worldline superconformal algebra for the superparticle remains an open problem.

\section{REFERENCES}

[1] W. Siegel, Phys. Lett.128B(1983)397; Class. Quantum Grav. 2 (1985) 302.

[2] E. Bergshoeff, R. Kallosh and A. Van Proeyen, Superparticle actions and gauge fixings, CERN preprint TH.6020/91 (to be published in Class. Quantum Grav.).

[3] D.P. Sorokin, V.I. Tkach and D.V. Volkov, Mod. Phys. Lett. A4 (1989) 901;

D.P. Sorokin, V.I. Tkach, D.V. Volkov and A.A. Zheltukhin, Phys. Lett. 216B (1989) 302.

[4] R. Brooks, F. Muhammad and S.J. Gates, Class. Quantum Grav. 3 (1986) 745;

J.Kowalski-Glikman, J.W. van Holten, S. Aoyama and J. Lukierski, Phys. Lett. 201B (1988) 487.

[5] P.S. Howe and P.K. Townsend, Phys. Lett. 259B (1991) 285.

[6] F. Delduc and E. Sokatchev, Superparticle with extended worldline superpsymmetry, preprint PAR-LPTHE/91-14 (to be published in Nucl. Phys. B).

[7] N. Berkovits, Phys. Lett. 247B(1990) 45.

[8] R. Penrose and W. Rindler, Spinors and space-time (Cambridge University Press, 1986).

[9] A. Galperin, E. Ivanov, S. Kalitzin, V. Ogievetsky and E. Sokatchev, Class. Quantum Grav. 1 (1984) 469.

[10] E. Witten, Nucl. Phys. B266 (1986) 245.

[11] E. Bergshoeff, P. Howe, C.N. Pope, E. Sezgin and E. Sokatchev, Nucl. Phys. B354 (1991) 113.

[12] P.S. Howe, Phys. Lett. 258B (1991) 141. 
[13] E. Bergshoeff, F. Delduc and E .Sokatchev, Phys. Lett. 262B (1991) 444; P.S. Howe, Pure spinors, function superspaces and supergravity theories in ten and eleven dimensions, Stony Brook preprint ITP-SB-91-18.

[14] E. Sokatchev, Phys. Lett. 169B (1986) 209; Class. Quantum Grav. 4 (1987) 237.

[15] E. Nissimov, S. Pacheva and S. Solomon, Nucl. Phys. B296 (1988) 462;

E. Nissimov, S. Pacheva and S. Solomon, Nucl. Phys. B297 (1988) 349;

R. Kallosh and M. Rahmanov, Phys. Lett. 209B (1988) 233.

[16] I. Gel'fand, M. Graev and N. Vilenkin, Generalized functions, v.5, (Academic Press, New York and London, 1966);

N. Vilenkin, Special functions and the theory of group representations (American Mathematical Society, Providence, 1968).

[17] I. Bengtsson and M. Cederwall, Nucl. Phys. B221 (1988) 81.

[18] P. Furlan and R. Raczka, J. Math. Phys. 26 (1985) 3021.

[19] T. Kugo and P. Townsend, Nucl. Phys. B221 (1983) 357.

[19] E.A. Ivanov and A.A. Kapustnikov, Towards a tensor calculus for $\kappa$-supersymmetry, preprint IC/91/68. 